\title{
Club good mechanisms: from free-riders to citizen- shareholders, from impossibility to characterization
}

Citation for published version (APA):

Mackenzie, A., \& Trudeau, C. (2018). Club good mechanisms: from free-riders to citizen-shareholders, from impossibility to characterization. Maastricht University, Graduate School of Business and Economics. GSBE Research Memoranda No. 012 https://doi.org/10.26481/umagsb.2018012

Document status and date:

Published: 08/05/2018

DOI:

10.26481/umagsb.2018012

Document Version:

Publisher's PDF, also known as Version of record

\section{Please check the document version of this publication:}

- A submitted manuscript is the version of the article upon submission and before peer-review. There can be important differences between the submitted version and the official published version of record.

People interested in the research are advised to contact the author for the final version of the publication, or visit the DOI to the publisher's website.

- The final author version and the galley proof are versions of the publication after peer review.

- The final published version features the final layout of the paper including the volume, issue and page numbers.

Link to publication

\footnotetext{
General rights rights.

- You may freely distribute the URL identifying the publication in the public portal. please follow below link for the End User Agreement:

www.umlib.nl/taverne-license

Take down policy

If you believe that this document breaches copyright please contact us at:

repository@maastrichtuniversity.nl

providing details and we will investigate your claim.
}

Copyright and moral rights for the publications made accessible in the public portal are retained by the authors and/or other copyright owners and it is a condition of accessing publications that users recognise and abide by the legal requirements associated with these

- Users may download and print one copy of any publication from the public portal for the purpose of private study or research.

- You may not further distribute the material or use it for any profit-making activity or commercial gain

If the publication is distributed under the terms of Article $25 \mathrm{fa}$ of the Dutch Copyright Act, indicated by the "Taverne" license above, 
Andrew Mackenzie,

Christian Trudeau

Club good mechanisms: from free-riders to citizenshareholders, from impossibility to characterization

$\mathrm{RM} / 18 / 012$

\section{GSBE}

Maastricht University School of Business and Economics

Graduate School of Business and Economics

P.O Box 616

NL-6200 MD Maastricht

The Netherlands 


\title{
Club GOOd MeChanisms: FROM FREE-RIDERS TO CITIZEN-SHAREHOLDERS, FROM IMPOSSIBILITY TO CHARACTERIZATION
}

\author{
Andrew Mackenzie* And Christian Trudeau ${ }^{\dagger \dagger}$ \\ This draft: January 18, 2017
}

\begin{abstract}
Consider a community that shares a technology for producing a club good (Buchanan, 1965): any group of agents can "win" for an associated monetary cost. Who should win, and how should production be funded? To address this question, we seek rules (that is, direct mechanisms) where each agent participates voluntarily and is incentivized to report his valuation honestly, and where these reports are used to select winners efficiently without running a deficit.

We find that whether or not there are such rules depends on the production technology. If costs are even "somewhat concave," then there are no such rules: the free-rider problem (Wicksell, 1896; Samuelson, 1954; Green and Laffont, 1979) persists even when agents who do not contribute can be excluded. If costs are symmetric and convex, however, then there are such rules that moreover satisfy no-envy-in-trades (Kolm, 1971; Schmeidler and Vind, 1972). We characterize this class, whose Pareto-worst member is the familiar minimum-price Walrasian rule (Vickrey, 1961; Clarke, 1971; Groves, 1973; Demange, 1982; Leonard, 1983); the other rules do better by treating the agents as equal shareholders in the technology and offering social dividends (Lange, 1936).
\end{abstract}

\section{Introduction}

\subsection{Executive summary}

According to one version of the free-rider problem (Wicksell, 1896; Samuelson, 1954; Green and Laffont, 1979), a community seeking to efficiently provide a public good on the basis of reported interest, with production funded by voluntary contributions, is doomed to fail. In the usual story, an agent inevitably under-reports his interest and

\footnotetext{
*Department of Economics, University of Rochester, Rochester, NY, 14627, USA. Email: amacken2@ur.rochester.edu

${ }^{\dagger}$ Department of Economics, University of Windsor 401 Sunset Avenue, Windsor, Ontario, Canada. Email: trudeauc@uwindsor.ca

${ }^{\ddagger}$ We thank Yu Awaya, Eddie Dekel, Hervé Moulin, Yves Sprumont, and Rodrigo Velez; seminar participants at University of Glasgow, University of St Andrews, the 2015 Midwest Theory Conference in Rochester, and the 2016 International Conference on Game Theory in Stony Brook; and especially William Thomson.
} 
underpays, only to later reap benefit from whatever is produced, thereby free-riding on the contributions of his peers. But might such a person confess his true valuation upon learning that, should he inadequately contribute, he can and will be excluded from the final product?

In this article, we consider the problem of providing a club good (Buchanan, 1965): a good that is (i) nonrival, like a public good, in that no agent's consumption impacts another's (positively or negatively), and (ii) excludable, like a private good, in that any agent can be prevented from consuming it. Examples include gated parks, high-speed internet cables, libraries with membership, city sewage, and the like. We find that the ability to exclude non-contributors helps to resolve the free-rider problem sometimes, but not always. In particular, when costs are even "somewhat" concave, the problem unfortunately persists. On the other hand, when costs are symmetric and convex, we identify a rich new family of mechanisms that improve on the familiar minimum price Walrasian rule (Vickrey, 1961; Clarke, 1971; Groves, 1973; Demange, 1982; Leonard, 1983) by treating members of the community as shareholders in the production process.

In our model, any group of winners can be provided the club good for an associated monetary cost, but each agent's valuation of winning is private information. Because efficient provision requires the agents' collective information, we investigate designing an institution called a game form, or mechanism, which specifies an unambiguous procedure by which the agents make choices to ultimately determine their outcome. So that these choices plausibly lead to desired outcomes, we require that no matter the agents' true preferences, a desired outcome is a dominant strategy equilibrium.

By the revelation principle (Hurwicz, 1972; Gibbard, 1973), it is without loss of generality to restrict attention to direct mechanisms, or rules, where the agents simultaneously report their valuations, then these reports determine who wins and how production is funded. Our objective is to identify rules that satisfy the following requirements:

- no-deficit: production is funded entirely by the contributions of the agents,

- production efficiency: the winning group is compatible with Pareto-efficiency (though money may be burned) ${ }^{1}$

- voluntarism: no agent prefers losing without a transfer to his assignment, and

- strategy-proofness: honesty is a dominant strategy for each agent.

Whether or not these objectives are compatible depends on the production technology, which follows from the literature:

1. When serving nobody is free, serving everybody has some finite positive cost, and serving any other group has infinite cost, we are essentially providing a pure public good, where the Groves rules (Groves, 1973) are the only ones satisfying production efficiency and strategy-proofness (Green and Laffont, 1977; Holmström, 1979), and the voluntary ones necessarily run a deficit (Green and Laffont, 1979).

2. When serving up to $k$ winners is free, while serving more winners has infinite cost, we are essentially distributing $k$ identical indivisible objects, where it is well-known that the Vickrey rule (Vickrey, 1961) satisfies our criteria.

\footnotetext{
${ }^{1}$ This requirement is sometimes called allocative efficiency, decision efficiency, or partial efficiency. Because agents have quasi-linear preferences in our setting, this axiom is equivalent to the requirement that the group of winners is selected to maximize Marshallian surplus.
} 


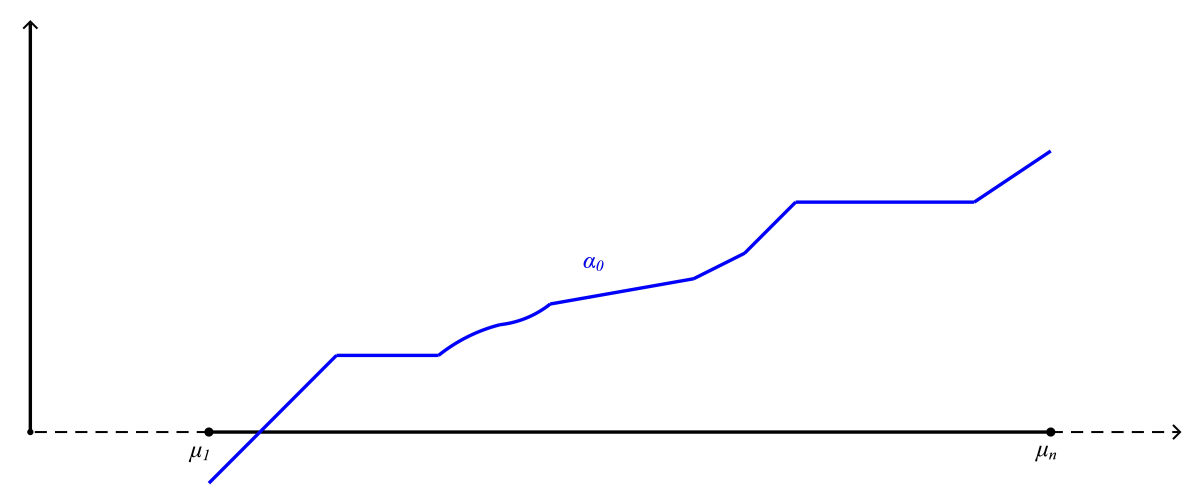

Figure 1: An example of a social-dividend schedule, $\alpha_{0}$. Here, $\mu_{1}$ is the marginal cost of producing the first object, while $\mu_{n}$ is the marginal cost of producing the $n$ th. The schedule is a Lipschitz-continuous function over this interval such that the slope between any two points on the curve is between 0 and 1 . Each agent faces a price in this interval, which is determined by a particular function that anonymously processes the bids of his peers. The point on the curve associated with this price is his social-dividend award, and strategically, the agent can choose whether to lose with the award or win with the award minus the price.

Our contribution is to extend both of these observations to our general club good model.

Theorem 1 is our strong statement of the free-rider problem: if no agents can be served for free, and if there are weak returns to scale in the sense that some group's cost is less than the sum of costs for its members individually, then no rule satisfies our requirements. Unfortunately, this applies to all subadditive cost functions, which are the production technologies that lead naturally to monopoly (Baumol, 1977). This is disappointing, because we might have hoped that mechanisms could help improve market outcomes in these industries.

On the other hand, if costs are finite, symmetric, and convex, then there are rules that not only meet our criteria, but moreover satisfy no-envy-in-trades (Kolm, 1971; Schmeidler and Vind, 1972), the equal-opportunity requirement that no agent prefers another's net change in resources to his own. Theorem 2 characterizes the rich class of rules satisfying production efficiency, strategy-proofness, and no-envy-in-trades, whose rules each use the same price function to offer a price to each agent by processing the bids of his peers anonymously. Each rule additionally has its own social-dividend schedule, which offers each agent a social-dividend award determined solely by his price. Strategically, each agent either (i) loses with this award, which is the outcome when he bids less than his price, or (ii) wins for this award minus his price, which is the outcome when he bids more than his price. Moreover, the social-dividend schedule is a nondecreasing Lipschitzcontinuous function of price, and the slope between any two points on its curve is at most 1 (see Figure 1). We refer to rules in this class as citizen-shareholder rules.

Theorem 3 characterizes the citizen-shareholder rules that satisfy no-deficit, which we call cost-constrained citizen-shareholder rules, using a finite list of constraints on the social-dividend schedule. Theorem 4 identifies the rules that are Pareto-undominated in this class, as well as the rules that are Pareto-undominated in its voluntary subclass. 


\subsection{Example: The Big Show}

When costs are finite, symmetric, and convex, there is in general a large class of Paretoundominated rules in our class, but in the special case that there are three agents, there is an essentially-unique Pareto-dominant rule. We illustrate this rule with an example.

Three fans want to go to some event, which we call the Big Show, but are disappointed to learn that it is already sold out. Undeterred, they go online to search for tickets that are being re-sold, and sure enough they find a website where there are three scalpers, each selling one ticket. Each of the fans is willing to pay $\$ 30$, while the scalpers' prices are $\$ 10, \$ 20$, and $\$ 80$.

This is an economy with indivisible objects and money, where each agent wants at most one object, and where each agent is endowed with at most one object, as in the classic horse market economy (Böhm-Bawerk, 1888; Shapley and Shubik, 1972); we describe our contribution to this literature in the conclusion. Here, we simply consider what happens in our Big Show economy, focusing specifically on the outcomes of the fans. Their outcomes, of course, depend on the institutional arrangements that prevail in society, of which we consider four possibilities:

- Jungle: ${ }^{2}$ First, suppose that each ticket can be acquired with the click of a button at the scalper's listed price (as in eBay's Buy It Now prices), and the fans race one another to acquire tickets.

Outcome: The fastest-clicker receives a ticket and pays $\$ 10$, the second-fastestclicker receives a ticket and pays $\$ 20$, and the slowest-clicker stays home and receives no transfer.

- Walrasian market (Walras, 1896): Suppose now that the scalper's listed prices are reservation prices, which we might indeed see if the scalpers are trying to maximize revenue (Myerson, 1981). The fans go on to offer competing bids on each ticket. At Walrasian equilibrium, there is a Walrasian price at which supply equals demand, which must be $\$ 30$ : at a lower price, three fans demand while fewer scalpers supply; at a higher price, no fans demand while some scalper supplies.

Outcome: Two fans receive a ticket and pay $\$ 30$, while the third fan stays home and receives no transfer.

- Monopoly: Suppose now that some outside party acquires ownership of the website, so that the three fans cannot directly access the scalpers, but must go through the owner. The owner views his website as a technology for producing tickets (by paying the scalpers as needed). Moreover, the owner has a monopoly on tickets, and wishes to sell to the three fans in order to maximize profit. Whether or not the owner is able to price-discriminate, two fans pay their full valuations while no ticket is sold to the third fan.

Outcome: Same as for the Walrasian market.

- OUR RUlE: Finally, suppose that the three fans seek the help of a mediator. The mediator does not know their valuations, so he commits to using our rule and asks each fan how much he is willing to pay. It is a dominant strategy for each to report

${ }^{2}$ For lack of a better term, we borrow jungle from Piccione and Rubinstein (2007), though our notion differs from theirs in that we do not consider a stronger fan appropriating money from a weaker fan. 


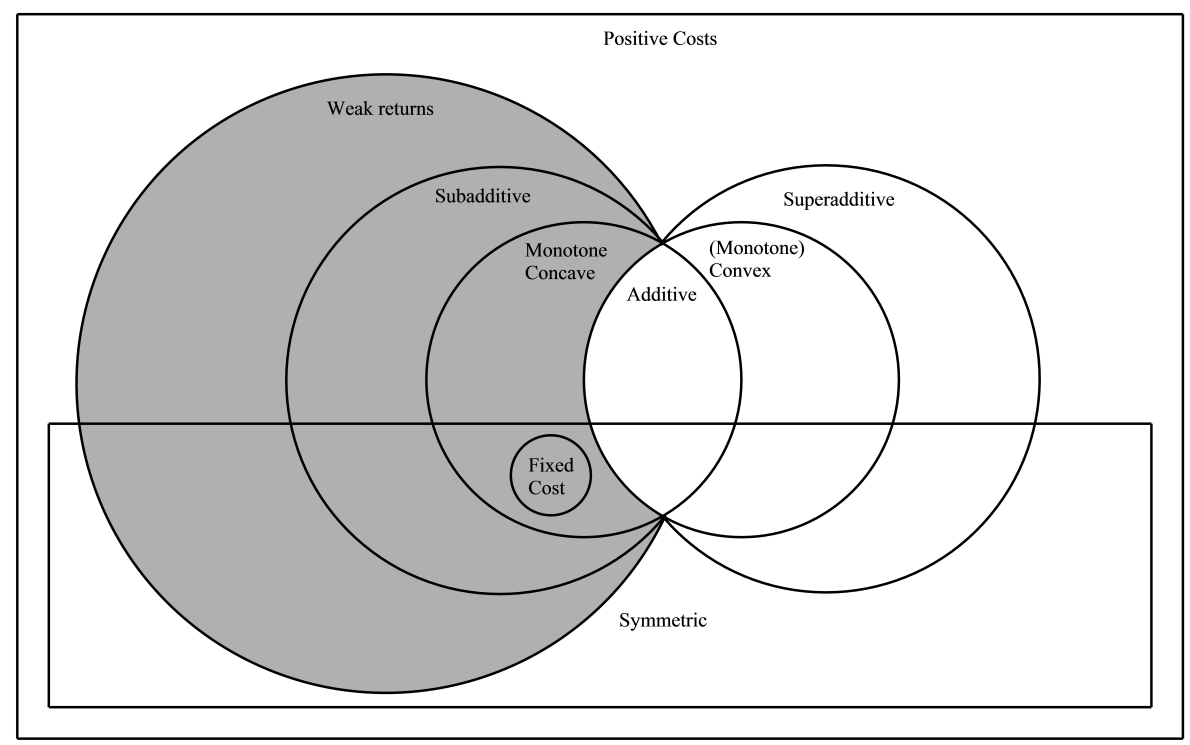

Figure 2: Logical relations between standard domains of cost functions. Theorem 1 states that there is no rule satisfying our requirements when the cost function is in the shaded region.

the truth, and in order to form our prediction we assume that the truth is indeed reported.

Outcome: Two fans receive a ticket and pay $\$ 20$, while the third fan stays home and receives $\$ 10$ as consolation.

It is straightforward to verify that each fan prefers our rule's outcome to both the Walrasian market outcome and the monopoly outcome. With respect to the jungle, our rule transfers $\$ 10$ from the fastest-clicker to the slowest-clicker, removing envy derived from clicking speed. Whether or not one prefers the jungle to our rule may depend on one's own clicking speed, or whether or not one views clicking speed as a virtue that should help determine where our institutions direct resources; we simply remark that this trait is not related to valuation or contribution.

When the scalpers are considered, our rule does not offer a Pareto-improvement over the Walrasian outcome, but rather a transfer from the scalpers to the fans. That said, it is easy to see that our analysis is legitimate if the scalpers are replaced by a technology for producing tickets, and here our rule indeed offers a Pareto-improvement over the Walrasian outcome.

For this example, our rule does not burn money: the two fans who go to the show together spend $\$ 40$, of which $\$ 10$ goes to the first scalper, $\$ 20$ goes to the second scalper, and $\$ 10$ goes to the third fan. That said, our rule sometimes burns money upon receiving reports in order to remain compatible with incentives.

\subsection{Related literature}

Our model is a special case of the Green and Laffont (1979) model: the public decisions are specifically groups of winners, and preferences are restricted to reflect this additional structure. This preference restriction does not obstruct us from a fundamental result 
(Holmström, 1979) that the rules satisfying production efficiency and strategy-proofness are precisely the Groves rules (Groves, 1973). ${ }^{3}$

In turn, special cases of our model, given by particular classes of cost functions, have been previously studied (see Figure 2). In addition to the well-studied cases described earlier, there is axiomatic analysis for:

- the "almost-public" good class, where all nonempty groups share a fixed finite cost (Deb and Razzolini, 1999a; Deb and Razzolini, 1999b; Ohseto, 2000; Ohseto, 2005; Yu, 2007; Ohseto, 2009; Massó, Nicolò, Sen, Sharma, and Ülkü, 2015; Hashimoto and Saitoh, 2016),

- the monotone and concave class (Moulin and Shenker, 2001; Mutuswami, 2004), and

- the general case (Juarez, 2013; Juarez, working); these papers consider group strategyproofness.

With the exception of Moulin and Shenker (2001), however, these articles do not consider production efficiency; they usually instead consider budget balance, the requirement that there is no deficit and no money is burned. Together, production efficiency and budget balance are equivalent to Pareto-efficiency, and we cannot have both: Groves rules necessarily burn money to provide incentives (Green and Laffont, 1979).

For monotone and concave cost functions, Moulin and Shenker (2001) characterize the class of rules that satisfy production efficiency, voluntarism, strategy-proofness, and the additional requirement that no agent receives a positive transfer, which is fittingly called no positive transfers. An immediate corollary is that no such rule satisfies no-deficit; thus for these cost functions, our objectives are incompatible with no positive transfers. While we do not view no positive transfers as a normatively appealing objective, because it is a sort of welfare upper bound, this axiom can alternatively be interpreted as the physical restriction that no agent can transfer the divisible good to another. This could be the case, for example, if the divisible good is leisure instead of money.

Among our citizen-shareholder rules, the zero-dividend rule is the only rule that satisfies voluntarism and no positive transfers, and this rule moreover satisfies no deficit. This particular rule is in fact none other than the latest incarnation of an idea, known now by many names, that regularly emerges in these kinds of models. With public goods, it is called Clarke's pivot rule (Clarke, 1971; Green and Laffont, 1979; Moulin, 1986; Moulin and Shenker, 2001). When there is a social endowment of identical indivisible objects of which each agent desires at most one, it is called Vickrey's rule (Vickrey, 1961; Ohseto, 2006; Chew and Serizawa, 2007; Saitoh and Serizawa, 2008; Sakai, 2008; Ashlagi and Serizawa, 2012). More generally, this is the minimum-price Walrasian rule. ${ }^{4}$ Perhaps

\footnotetext{
${ }^{3}$ Technically, the theorem of Holmström (1979) does not directly apply because it considers maximizing the sum of valuations, while we take into account the cost function as in Roberts (1979) and maximize the Marshallian surplus. That said, this is only a superficial difference: the original proof goes through with this small modification line-by-line.

${ }^{4}$ When there are (possibly distinct) indivisible objects of which each agent desires at most one and preferences are quasi-linear, the Walrasian price vectors form a bounded lattice (Shapley and Shubik, 1972), and the minimum-price Walrasian rule, which assigns an outcome associated with the minimum Walrasian price vector, is strategy-proof (Demange, 1982; Leonard, 1983). These facts persist when preferences are not quasi-linear (Demange and Gale, 1985), and this rule was recently characterized (Morimoto and Serizawa, 2015). In fact, even when agents may consume several objects, Walrasian
} 
surprisingly, the zero-dividend rule is distinguished in another way among the citizenshareholder rules satisfying no-deficit and voluntarism: it is the Pareto-worst! Thus for this class, the content of no positive transfers is anti-efficiency. The welfare improvements offered by positive transfers have not gone unnoticed; for example, Guo, Markakis, Apt, and Conitzer (2013) study the Pareto-undominated Groves rules in the general Green and Laffont (1979) model.

Groves rules have not only been investigated on the basis of efficiency; fairness has been focal as well. The article contributes to the large literature on fair Groves rules for economies with indivisible objects and money, where:

- no-envy (Tinbergen, 1946; Foley, 1967) has been investigated (Pápai, 2003; Ohseto, 2006; Yengin, 2012b; Yengin, 2016),

- population monotonicity (Thomson, 1983) has been investigated (Yengin, 2013a),

- egalitarian-equivalence (Pazner and Schmeidler, 1978) has been investigated (Ohseto, 2004; Yengin, 2012a; Yengin, 2012b; Yengin, 2016),

- the identical-preferences-lower-bound (Steinhaus, 1948) has been investigated (Yengin, 2013b), and

- a parametric notion of maximizing the welfare of the worst-off agent (Rawls, 1971; Porter, Shoham, and Tennenholtz, 2004) has been investigated (Atlamaz and Yengin, 2008).

We consider no-envy-in-trades (Schmeidler and Vind, 1972), which mathematically plays the same role as no-envy in the above works; we make the distinction to emphasize a conceptual point. ${ }^{5}$ Our contribution to this literature is allowing production. Of these works, the most closely related is Ohseto (2006), which characterizes the strategy-proof and envy-free rules when there is a fixed supply of identical indivisible objects that all must be consumed. In this setting, no-envy implies Pareto-efficiency (Svensson, 1983), so mathematically both proofs involve the same axioms. In fact, one of the cases in our proof of the Consolation Lemma, which is used to prove Theorem 2, uses an adaptation of an argument from Ohseto (2006) (though the other case in our proof uses a novel argument). That said, we do not directly generalize Ohseto (2006), because we cannot simply add the restriction that all objects must be consumed as an axiom; the restriction also has implications for what is feasible, and therefore for whether or not a given allocation is Pareto-dominated.

Our work is related in spirit to early contributions to the free-rider problem, which often considered a single divisible public good. One of the focal rules in this setting is

prices form a lattice under reasonable assumptions: Gul and Stacchetti (1999) show that Walrasian prices (for objects, not bundles) form a lattice if each agent's preferences satisfy single improvement, which for monotonic preferences is equivalent to the gross substitutes condition of Kelso and Crawford (1982).

${ }^{5}$ In particular, no-envy requires that no agent prefers what another agent consumes to what he himself does, while no-envy-in-trades requires that no agent prefers another's net change in resources to his own. Both axioms play the same role mathematically because (i) we suppress private endowments in our notation, and (ii) the axioms are equivalent when all agents happen to have the same endowment. Conceptually, we allow envy due to the suppressed endowments, but forbid envy due to opportunities afforded by the mechanism. In our model, when production is unavailable, a pure redistribution of money could be necessary under no-envy, but violates no-envy-in-trades; in this way our fairness axiom takes property rights into account. 
the Lindahl rule (Lindahl, 1919), where one's contribution is based on marginal benefit, and before mechanism design was formalized, Samuelson (1954) argued that this rule is manipulable. In one of the seminal contributions to mechanism design, Hurwicz (1972) showed that such manipulability is not only a problem for public goods, ${ }^{6}$ and an analogous result for public goods (applying to all rules, not just the Lindahl rule) was proved shortly thereafter (Ledyard and Roberts, 1975; Groves and Ledyard, 1987).

Finally, we mention that in production economies with private goods, there are efficient and strategy-proof rules only in the extraordinary circumstance that production is linear, where each agent can operate the technology on his own without impacting his peers (Maniquet and Sprumont, 1999; Leroux, 2004). One might worry that the analogous result holds for club goods: there are rules satisfying our axioms only when the cost function is additive. Fortunately, however, the situation is not quite so bleak in our model.

In Section 2, we introduce the general model, the axioms, and the two special cases we study. In Section 3, we consider "somewhat concave" cost functions, and describe and discuss Theorem 1, our impossibility result. In Section 4, we consider the production of identical indivisible objects, where we describe and discuss a series of results culminating in Theorem 2, Theorem 3, and Theorem 4. Section 5 concludes.

\section{Model}

\subsection{General setting}

We consider a community of agents $N \equiv\{1,2, \ldots, n\}$ who share a production technology. Initially there is only one good, money, which is infinitely-divisible and homogeneous, and which may be transferred among agents; as the initial distribution of money has no impact on our analysis, we speak of agents consuming monetary transfers in order to simplify notation. ${ }^{7}$ The technology takes money as its input in order to create a group of winners, whose number we often denote by $w$. We call an agent who does not win a loser.

Each agent $i$ is selfish, caring only about his personal consumption of money and whether or not he wins. ${ }^{8}$ More precisely, $i$ has a preference relation $R_{i}$ over $\mathbb{R} \times\{0,1\}$, where the first coordinate specifies his monetary transfer and the second coordinate indicates whether or not he wins, with 1 representing that he does. Moreover, we assume that $R_{i}$ is strictly monotonic in money, quasi-linear in money, and that it satisfies the

${ }^{6}$ In particular, Hurwicz (1972) considered a two-agent economy with two private goods (that is, an Edgeworth box economy), and proved that every voluntary and efficient rule is manipulable. This disappointing result was generalized to the case where there are $n$ agents and $m$ goods (Serizawa, 2002), and in fact, the situation is even worse than that: no efficient and strategy-proof rule can guarantee that every agent receives an assignment that is bounded away from the origin (Serizawa and Weymark, 2003).

${ }^{7}$ Our analysis does not depend on the initial distribution of money for two reasons: (1) our assumptions imply that each agent's willingness to pay for an object is independent of his endowment, and (2) none of our axioms make reference to endowment. In fact, our approach implicitly assumes that addressing inequality is not a primary objective, since both property rights and fairness - as expressed by our axioms - are violated whenever money is redistributed and nothing is produced.

${ }^{8}$ The selfishness assumption restrict the possible interpretations of the club good-for example, it is not a telephone network (which is more valuable as more friends consume it) or a road (which is less valuable as traffic grows heavier). Notably, Velez (2016) recently considered a more general preference domain where agents may have other-regarding preferences. 
possibility-of-compensation assumption: there is an amount of money $v_{i}$ such that there is indifference between $\left(v_{i}, 0\right)$ and $(0,1)$. These assumptions together imply there is a unique valuation $v_{i} \in \mathbb{R}$ such that for each pair $\left(t_{i}, x_{i}\right),\left(t_{i}^{\prime}, x_{i}^{\prime}\right) \in \mathbb{R} \times\{0,1\}$,

$$
\left(t_{i}, x_{i}\right) R_{i}\left(t_{i}^{\prime}, x_{i}^{\prime}\right) \text { if and only if } v_{i} x_{i}+t_{i} \geq v_{i} x_{i}^{\prime}+t_{i}^{\prime} .
$$

Abusing notation, we identify each agent's preference relation by its valuation $v_{i}$. We use $V_{i} \subseteq \mathbb{R}$ to denote the class of admissible preference relations for $i$, and we use $V \equiv \times V_{i}$ to denote the class of admissible preference profiles. In both special settings we consider, agents share a common class of admissible preference profiles (that is, for each pair $i, j \in N, V_{i}=V_{j}$ ); our notation simply allows us to improve clarity.

The agents wish to select an allocation $(t, W) \in \mathbb{R}^{N} \times 2^{N}$, which specifies both a list of monetary transfers $t$ and a group of winners $W$. An allocation does not run a deficit if the winning group is produced using a sufficient amount of money as input. This is expressed using an exogenous cost function $C: 2^{N} \rightarrow \mathbb{R}_{+} \cup\{\infty\}$, with $C(\emptyset)=0$, which specifies the cost of production for each winning group.

Though an allocation that is favorable to the agents should be selected, preferences are private information. We consider the direct mechanism approach to this problem: each agent reports his preferences, then these reports jointly determine the allocation. Because we are interested in dominant strategy equilibria, focusing on direct mechanisms is without loss of generality (Hurwicz, 1972; Gibbard, 1973). Our problem is to recommend a direct mechanism, or a rule:

Definition: A transfer policy is a mapping $\tau: V \rightarrow \mathbb{R}^{N}$. A winner policy is a mapping $\varphi: V \rightarrow 2^{N}$. A rule $(\tau, \varphi)$ consists of a transfer policy $\tau$ and a winner policy $\varphi$.

Given a winner policy $\varphi$ and an agent $i$, we sometimes speak of the associated policy for determining whether or not $i$ is a winner, using $\varphi_{i}: V \rightarrow\{0,1\}$ for the mapping

$$
\varphi_{i}(v) \equiv \begin{cases}1, & i \in \varphi(v) \\ 0, & i \notin \varphi(v)\end{cases}
$$

In this article, we seek rules that satisfy desirable properties, or axioms.

\subsection{Axioms}

An axiom is a mathematical property of a rule that captures some aspect of our intuition. Each of the axioms we consider in this article is standard in the literature.

Our first axiom is the feasibility requirement that production is funded entirely by the agents:

No-DEFICIT: For each $v \in V$,

$$
0 \geq \sum \tau_{i}(v)+C(\varphi(v))
$$

Our second axiom is an efficiency notion which is weaker than Pareto-efficiency as it allows money to be burned. Informally: for a no-deficit allocation $(t, W)$, the money burned $m \geq 0$ solves $0=\sum t_{i}+C(W)+m$. The requirement is that if $(t, W)$ is selected, and before anything is produced $m$ units of money are burned and the no-deficit constraint is updated, then there should be no allocation $\left(t^{\prime}, W^{\prime}\right)$ that is still no-deficit, 
that everybody finds at least as desirable as $(t, W)$, and that one agent prefers to $(t, W)$. This can be seen as an ex-post notion: though certain resources may be destroyed, thereafter the allocation is Pareto-efficient even if production is reversible.

Because preferences are quasi-linear, the amount of money burned has no impact on whether or not this axiom is satisfied. Moreover, this notion is equivalent to selecting a group of winners that maximizes a particular objective function: the aggregate consumer surplus, or the sum across agents of the difference between willingness-to-pay and payment (Marshall, 1890), that is attainable if no money is burned. For convenience, we take this as our definition.

Formally, for each $v \in V$, define the (attainable Marshallian consumer) surplus $\sigma_{v}$ : $2^{N} \rightarrow \mathbb{R}$ by:

$$
\sigma_{v}(W) \equiv \sum_{i \in W} v_{i}-C(W)
$$

Production efficiency: For each $v \in V$ and each $W \subseteq N$,

$$
\sigma_{v}(\varphi(v)) \geq \sigma_{v}(W)
$$

Our third axiom asserts that a certain notion of property rights should be respected. In particular, without participating in the community and using the production technology, an agent could receive zero transfer and lose; the axiom requires that this is not preferred to what is received through participation. In other words, the rule should not be coercive:

Voluntarism: ${ }^{9}$ For each $i \in N$ and each $v \in V$,

$$
v_{i} \varphi_{i}(v)+\tau_{i}(v) \geq 0
$$

Each rule takes as input preference reports, but our normative axioms are only compelling when stated in terms of true preferences. Our justification for conflating the reports with the truth is our fourth axiom, which requires that it is a dominant strategy for each agent to report his valuation honestly: ${ }^{10}$

Strategy-PRoofness: For each $i \in N$, each $v \in V$, and each $v_{i}^{\prime} \in V_{i}$,

$$
v_{i} \varphi_{i}(v)+\tau_{i}(v) \geq v_{i} \varphi_{i}\left(v_{i}^{\prime}, v_{-i}\right)+\tau_{i}\left(v_{i}^{\prime}, v_{-i}\right) .
$$

Our fifth axiom is the equal-opportunity requirement that no agent prefers another's net change in resources to his own (Kolm, 1971; Schmeidler and Vind, 1972):

No-EnVy-IN-TRADES: For each pair $i, j \in N$ and each $v \in V$,

$$
v_{i} \varphi_{i}(v)+\tau_{i}(v) \geq v_{i} \varphi_{j}(v)+\tau_{j}(v) .
$$

\footnotetext{
${ }^{9}$ This is sometimes called individual rationality, which is appropriate when agents are in fact free to not participate.

${ }^{10}$ This standard justification for assuming honest reporting is unfortunately not well-supported by experimental evidence (Kagel, Harstad, and Levin, 1987; Kagel and Levin, 1993; Attiyeh, Franciosi, and Isaac, 2000; Kawagoe and Mori, 2001). This has motivated stronger implementation notions, such as secure implementation (Saijo, Sjöström, and Yamato, 2007) and obvious strategy-proofness (Li, 2016), which we do not consider here.
} 
Finally, we consider the standard incomplete ordering of rules in which a first rule dominates a second if, at each profile, the recommendation of the first Pareto-dominates the recommendation of the second:

Definition: For each pair of rules $\left(\tau^{*}, \varphi^{*}\right),(\tau, \varphi)$, we say $\left(\tau^{*}, \varphi^{*}\right)$ weakly Pareto-dominates $(\tau, \varphi)$ if for each $i \in N$ and each $v \in V$,

$$
v_{i} \varphi_{i}^{*}(v)+\tau_{i}^{*}(v) \geq v_{i} \varphi_{i}(v)+\tau_{i}(v) .
$$

If $\left(\tau^{*}, \varphi^{*}\right)$ weakly Pareto-dominates $(\tau, \varphi)$ but $(\tau, \varphi)$ does not weakly Pareto-dominate $\left(\tau^{*}, \varphi^{*}\right)$, then we say $\left(\tau^{*}, \varphi^{*}\right)$ (strictly) Pareto-dominates $(\tau, \varphi)$. If $\left(\tau^{*}, \varphi^{*}\right)$ and $(\tau, \varphi)$ weakly Pareto-dominate each other, then we say they are Pareto-indifferent.

\section{$3 \quad$ The service setting}

Our analysis focuses on two special cases of the general model, each of which involves additional assumptions about the structure of the cost function and the domain of admissible preferences.

In this section, we consider the service setting: no agents can be served for free, and there are weak returns to scale in the sense that some group's cost is less than the sum of costs for its members individually (see Figure 2 in introduction). Beyond these assumptions, the club good is not required to actually be a service; we simply use service to concisely contrast this setting from our second setting, and to suggest a few possible applications (such as a public utility or an exclusive postal service). The incompatibility of our axioms holds even if it is common knowledge that the service is undesirable, and strategic agents are unable to report otherwise:

Definition: The service setting entails the following assumptions:

- Positive costs: For each nonempty $W \subseteq N, C(W)>0$.

- Weak Returns: There is $W \subseteq N$ such that $\sum_{W} C(\{i\})>C(W)$.

- Desirable domain: For each $i \in N, V_{i}=\mathbb{R}_{+}$.

As discussed in the introduction, when serving nobody is free, serving everybody has some finite positive cost, and serving any other group has infinite cost, no rule satisfies our axioms (Groves, 1973; Green and Laffont, 1977; Holmström, 1979; Green and Laffont, 1979). On the other hand, when costs are positive and concave, our axioms are incompatible when the divisible resource cannot be transferred from one agent to another (Moulin and Shenker, 2001).

Unfortunately, we find that these negative results are part of a broader, pervasive impossibility. The incompatibility of our axioms is not caused by non-excludability of the indivisible output, nor by non-transferability of the divisible input, and moreover this incompatibility holds across a large class of cost functions:

Theorem 1: In the service setting, there is no rule satisfying no-deficit, production efficiency, voluntarism, and strategy-proofness. 
The proof is in Appendix 1. For these cost functions, the free-rider problem cannot be solved by excluding non-contributors: any direct mechanism is either unaffordable, inefficient, manipulable, or coercive.

We remark that this impossibility is tight. If any of the four axioms is dropped, there is a rule that satisfies the other three:

- No-Deficit: For each $v \in V$ and each $i \in N$, define $\sigma_{v}^{*} \equiv \max _{W \subseteq N} \sigma_{v}(W)$ to be the maximal surplus, and define $\sigma_{v}^{-i} \equiv \max _{W \subseteq N \backslash\{i\}} \sigma_{v}(W)$ to be the maximal surplus among groups that do not include $i$. Let $\varphi^{*}(v)$ be such that $\sigma_{v}\left(\varphi^{*}(v)\right)=\sigma_{v}^{*}$, and for each $i \in N$, define $\tau_{i}^{1}(v) \equiv-v_{i} \varphi_{i}^{*}(v)+\left(\sigma_{v}^{*}-\sigma_{v}^{-i}\right)$. Then $\left(\tau^{1}, \varphi^{*}\right)$ is (welfareequivalent to) the marginal cost pricing mechanism of Moulin and Shenker (2001), and therefore satisfies production efficiency, voluntarism, and strategy-proofness, but fails no-deficit.

- Production efficiency: For each $v \in V$ and each $i \in N$, define $\varphi^{2}(v) \equiv \emptyset$ and define $\tau^{2}(v) \equiv 0$. It is easy to see that $\left(\tau^{2}, \varphi^{2}\right)$ satisfies no-deficit, voluntarism, and strategy-proofness, but fails production efficiency.

- Voluntarism: Let $\tau^{4}(v)$ be such that for each $i \in N, \tau_{i}^{4}(v)=-\max _{W \subseteq N} C(W)$. It is easy to see that $\left(\tau^{4}, \varphi^{*}\right)$ satisfies no-deficit, production efficiency, and strategyproofness, but fails voluntarism.

- Strategy-proofness: For each $i \in N$, define $\tau_{i}^{3}(v) \equiv-\varphi_{i}^{*}(v) v_{i}$. It is easy to see that $\left(\tau^{3}, \varphi^{*}\right)$ satisfies production efficiency and voluntarism, but fails strategy-proofness. To see that no-deficit is satisfied, assume, by way of contradiction, that there is $v \in V$ such that $\sum \tau_{i}^{3}(v)+C\left(\varphi^{*}(v)\right)>0$. Then $0>\sum_{\varphi^{*}(v)} v_{i}-C\left(\varphi^{*}(v)\right)$, so $\sigma_{v}(\emptyset)>\sigma_{v}\left(\varphi^{*}(v)\right)$, contradicting that $\varphi^{*}$ maximizes surplus at $v$.

Moreover, if either of the cost function restrictions is dropped, there is a rule satisfying all four axioms:

- Weak Returns: This is the topic of Section 4.

- Positive Costs: Consider $N=\{1,2\}$ and $C$ given by $C(\{1\})=0, C(\{2\})=2$ and $C(\{1,2\})=1$. Let $\varphi(v)=\{1,2\}$ if $v_{2} \geq 1$ and $\varphi(v)=\{1\}$ otherwise, and let $\tau(v)=(0,1)$ if $2 \in \varphi(v)$ and $\tau(v)=(0,0)$ otherwise. It is easy to see that $(\tau, \varphi)$ satisfies all four axioms.

\section{The object setting}

We move onward from the disappointing findings of Section 3, and now turn our attention to the object setting: costs are finite, the cost of serving a group depends only on its size (not on the specific identities of its members), and costs are convex. The simplest interpretation is that we are producing and distributing identical indivisible objects, and producing additional objects becomes progressively more expensive. The compatibility of our axioms holds even if any given agent might view the object as either a good or a bad:

DEFINITION: The object setting entails the following assumptions:

- Finite costs: For each $W \subseteq N, C(W)<\infty$. 
- Symmetry: For each pair $W, W^{\prime} \subseteq N,|W|=\left|W^{\prime}\right|$ implies $C(W)=C\left(W^{\prime}\right)$.

- Convexity: For each $i \in N$ and each pair $W, W^{\prime} \subseteq N \backslash\{i\}$ such that $W \subseteq W^{\prime}$,

$$
C\left(W^{\prime} \cup\{i\}\right)-C\left(W^{\prime}\right) \geq C(W \cup\{i\})-C(W) .
$$

- Full domain: For each $i \in N, V_{i}=\mathbb{R}$.

As costs are symmetric, we abuse notation, writing $C:\{0,1,2, \ldots, n\} \rightarrow \mathbb{R}_{+}$, where $C(w)$ denotes the monetary cost of producing $w$ winners. Moreover, as costs are finite and convex, there are marginal costs $\mu_{1}, \mu_{2}, \ldots, \mu_{n} \in \mathbb{R}_{+}$such that

(i) $\mu_{n} \geq \mu_{n-1} \geq \ldots \geq \mu_{1}$, and

(ii) for each $w \in\{1,2, \ldots, n\}, C(w)=\sum_{i=1}^{w} \mu_{i}$.

We begin by introducing an index that ranks agents from highest valuation to lowest, breaking ties using the original index: for each $v \in V$, define $A_{1}(v), A_{2}(v), \ldots, A_{n}(v) \in N$ such that (1) $\left\{A_{i}(v)\right\}=N$, and (2) for each pair $r, r^{\prime} \in\{1,2, \ldots, n\}$ such that $r^{\prime}>r$, either

(i) $v_{A_{r}(v)}>v_{A_{r^{\prime}}(v)}$, or

(ii) $v_{A_{r}(v)}=v_{A_{r^{\prime}}(v)}$ and $A_{r^{\prime}}(v)>A_{r}(v)$.

We say that $r$ is the rank-index of agent $A_{r}(v)$ at $v$. We also introduce an index for listing the valuations themselves from highest to lowest, thinking of them as "bids" when ranked: for each $v \in V$ and each $r \in\{1,2, \ldots, n\}$, define $B_{r}(v) \equiv v_{A_{r}(v)}$. We say that $r$ is the rank-index of valuation $B_{r}(v)$ at $v$. For emphasis, $B_{1}(v) \geq B_{2}(v) \geq \ldots \geq B_{n}(v)$.

Analogous notation is also used for each $i \in N$ and each $v_{-i} \in V_{-i}$. Put another way, $N \backslash\{i\}=\left\{A_{1}\left(v_{-i}\right), A_{2}\left(v_{-i}\right), \ldots, A_{n-1}\left(v_{-i}\right)\right\}, B_{1}\left(v_{-i}\right) \geq B_{2}\left(v_{-i}\right) \geq \ldots \geq B_{n-1}\left(v_{-i}\right)$, and the re-indexed agents correspond to the re-indexed values at $v_{-i}$ (with ties broken using the original index as above).

Our first result characterizes the rules satisfying production efficiency, whose defining feature is that the winners have the high valuations, justifying the term "bid" for preference report. More precisely, the winners are the high bidders, provided that each winning valuation is at least the marginal cost of the final object and each losing valuations does not exceed the marginal cost of another:

BIDDING LEMma: In the object setting, a rule $(\tau, \varphi)$ satisfies production efficiency if and only if for each $v \in V$ with $w \equiv|\varphi(v)|$,

(i) for each $i \in \varphi(v)$ and each $j \in N \backslash \varphi(v), v_{i} \geq v_{j}$,

(ii) $w \neq 0$ implies $B_{w}(v) \geq \mu_{w}$, and

(iii) $w \neq n$ implies $\mu_{w+1} \geq B_{w+1}(v)$.

The proof is in Appendix 2. By the Bidding Lemma, at each profile $v$, a rule satisfying production efficiency first selects a number of winners $w$ satisfying two constraints, then selects a group of that size whose valuations are highest. This is suggestive of an "equilibrium quantity," though there is no corresponding suggestion of an "equilibrium price."

Our second result characterizes the rules satisfying strategy-proofness, whose defining feature is that each agent's attainable set is described by a consolation award and a price. In particular, each agent can either lose for the consolation award or win for the 
consolation award minus the price. Moreover, the agent wins when he bids more than the price and loses when he bids less:

PRICE LEMma: In the object setting, a rule $(\tau, \varphi)$ satisfies strategy-proofness if and only if for each $i \in N$ and each $v_{-i} \in V_{-i}$, there are $\tau_{i}^{+}\left(v_{-i}\right), \tau_{i}^{-}\left(v_{-i}\right) \in \mathbb{R}$ and $p_{i}\left(v_{-i}\right) \in$ $\mathbb{R} \cup\{-\infty, \infty\}$ such that for each $v_{i} \in V_{i}$,

(i) $\left(\tau_{i}(v), \varphi_{i}(v)\right)$ is either $\left(\tau_{i}^{+}\left(v_{-i}\right), 1\right)$ or $\left(\tau_{i}^{-}\left(v_{-i}\right), 0\right)$,

(ii) $p_{i}\left(v_{-i}\right) \notin\{-\infty, \infty\}$ implies $\tau_{i}^{+}\left(v_{-i}\right)=\tau_{i}^{-}\left(v_{-i}\right)-p_{i}\left(v_{-i}\right)$,

(iii) $v_{i}>p_{i}\left(v_{-i}\right)$ implies $i \in \varphi(v)$, and

(iv) $p_{i}\left(v_{-i}\right)>v_{i}$ implies $i \notin \varphi(v)$.

The proof is in Appendix 3. Each agent faces a personalized price determined by the reports of his peers, though this is not quite enough to suggest an "equilibrium price."

Our next objective is to characterize the rules satisfying both production efficiency and strategy-proofness. As the winner policy is fixed, what remains is to describe the transfer policy. By the Price Lemma, each agent $i$ faces a price $p_{i}\left(v_{-i}\right)$ such that he demands an object with a higher report and demands no object with a lower report. For such a rule to satisfy production efficiency, these $n$ prices must form an "equilibrium" at which honest agents independently demand objects in a manner that maximizes surplus. This dramatically restricts the manner in which an agent's price can depend on the reports of his peers.

For each $i \in N$, define the price for $i, \pi: V_{-i} \rightarrow\left[\mu_{1}, \mu_{n}\right]$, by:

$$
\begin{aligned}
& \Omega\left(v_{-i}\right) \equiv\left|\left\{r \in\{1,2, \ldots, n-1\} \mid B_{r}\left(v_{-i}\right) \geq \mu_{r}\right\}\right|, \\
& \pi\left(v_{-i}\right) \equiv \begin{cases}\min \left\{B_{\Omega\left(v_{-i}\right)}\left(v_{-i}\right), \mu_{\Omega\left(v_{-i}\right)+1}\right\}, & \Omega\left(v_{-i}\right)>0, \\
\mu_{1}, & \Omega\left(v_{-i}\right)=0 .\end{cases}
\end{aligned}
$$

Thus $\Omega\left(v_{-i}\right)$ is the maximum number of $i$ 's peers that may win, and $\pi\left(v_{-i}\right)$ is either the lowest of the bids of these peers or the marginal cost of an additional object - whichever is lower. Note that $\pi$ is not indexed by any agent and that it processes reports anonymously; each agent's price is determined by this common function that only depends on the list of peer bids, but not on which peers submitted which bids.

We use "auction" to refer to a rule in which both (i) winners are high bidders, and (ii) each agent's bid is used to determine whether or not he demands an object at a particular price. The following class of auctions use prices, and are characterized by production efficiency and strategy-proofness:

Definition: For each $i \in N$, a peer-consolation schedule is a function $\alpha_{i}: V_{-i} \rightarrow \mathbb{R}$. A rule $(\tau, \varphi)$ is a peer-consolation rule if (I) it satisfies production efficiency, and (II) for each $i \in N$, there is a peer-consolation schedule $\alpha_{i}: V_{-i} \rightarrow \mathbb{R}$ such that for each $v_{-i} \in V_{-i}$,

(i) $\left(\tau_{i}(v), \varphi_{i}(v)\right)$ is either $\left(\alpha_{i}\left(v_{-i}\right)-\pi\left(v_{-i}\right), 1\right)$ or $\left(\alpha_{i}\left(v_{-i}\right), 0\right)$,

(ii) $v_{i}>\pi\left(v_{-i}\right)$ implies $i \in \varphi(v)$, and

(iii) $\pi\left(v_{-i}\right)>v_{i}$ implies $i \notin \varphi(v)$.

Auction Lemma: In the object setting, a rule $(\tau, \varphi)$ satisfies production efficiency and strategy-proofness if and only if it is a peer-consolation rule.

The proof is in Appendix 4. By a superficial adaptation of Holmström (1979) (see 
footnote 3), these are precisely the Groves (1973) rules; the additional structure in our representation is due to the additional structure in our model.

We have thus far emphasized that each agent's price is determined by the reports of his peers. But when the entire profile of reports is considered, the prices are collectively harmonious in a particular way: winners face one price and losers face another. Moreover, the lowest winning bid is at least the losers' price, which is at least the winners' price, which is at least the highest losing bid. Formally, define:

$$
\begin{gathered}
\Omega(v) \equiv\left|\left\{r \in\{1,2, \ldots, n\} \mid B_{r}(v) \geq \mu_{r}\right\}\right|, \\
\pi_{+}(v) \equiv\left\{\begin{array}{lr}
\mu_{n}, & \Omega(v)=n, \\
\max \left\{B_{\Omega(v)+1}(v), \mu_{\Omega(v)}\right\}, & n>\Omega(v)>0 \\
\mu_{1}, & \Omega(v)=0 .
\end{array}\right. \\
\pi_{-}(v) \equiv\left\{\begin{array}{lr}
\mu_{n}, & \Omega(v)=n, \\
\min \left\{B_{\Omega(v)}(v), \mu_{\Omega(v)+1}\right\}, & n>\Omega(v)>0 \\
\mu_{1}, & \Omega(v)=0 .
\end{array}\right.
\end{gathered}
$$

Winner-Loser Prices Lemma: In the object setting, for each $v \in V$ and each $i \in N$,

(i) $v_{i} \geq \pi\left(v_{-i}\right)$ implies $\pi\left(v_{-i}\right)=\pi_{+}(v)$, and

(ii) $\pi\left(v_{-i}\right) \geq v_{i}$ implies $\pi\left(v_{-i}\right)=\pi_{-}(v)$.

Moreover, either $v_{i} \geq \pi_{-}(v) \geq \pi_{+}(v)$ or $\pi_{-}(v) \geq \pi_{+}(v) \geq v_{i}$.

The proof is in Appendix 5.

Which peer-consolation rules satisfy no-envy-in-trades? In order to provide a complete answer, we first observe that no-envy-in-trades imposes a strong restriction on peerconsolation schedules: an agent's consolation award cannot depend on the reports of his peers in any way, but rather can only depend on a particular summary statistic of their reports. Moreover, this particular summary statistic is his own price:

Consolation Lemma: In the object setting, if a rule $(\tau, \varphi)$ satisfies production efficiency, strategy-proofness, and no-envy-in-trades, then $(\tau, \varphi)$ is a peer-consolation rule with peer-consolation schedules $\left(\alpha_{i}\right)$ such that for each $i \in N$ and each pair $v_{-i}, v_{-i}^{\prime} \in V_{-i}$,

$$
\pi\left(v_{-i}\right)=\pi\left(v_{-i}^{\prime}\right) \text { implies } \alpha_{i}\left(v_{-i}\right)=\alpha_{i}\left(v_{-i}^{\prime}\right) .
$$

The proof, which is the central proof of this article, is in Appendix 6 .

In fact, no-envy-in-trades further implies that agents share a common peer-consolation schedule that depends only on price, and moreover that this common schedule is nondecreasing, Lipschitz-continuous, and without a slope greater than 1 at any point. We call a communal peer-consolation schedule with these features a social-dividend schedule:

Definition: A social-dividend schedule is a (Lipschitz-continuous) function $\alpha_{0}:\left[\mu_{1}, \mu_{n}\right] \rightarrow$ $\mathbb{R}$ such that for each distinct pair $\pi^{\prime}, \pi \in\left[\mu_{1}, \mu_{n}\right]$,

$$
\frac{\alpha_{0}\left(\pi^{\prime}\right)-\alpha_{0}(\pi)}{\pi^{\prime}-\pi} \in[0,1] .
$$

A rule $(\tau, \varphi)$ is citizen-shareholder rule if (I) it satisfies production efficiency, and (II) there is a social-dividend schedule $\alpha_{0}$ such that for each $i \in N$ and each $v_{-i} \in V_{-i}$, 
(i) $\left(\tau_{i}(v), \varphi_{i}(v)\right)$ is either $\left(\alpha_{0}\left(\pi\left(v_{-i}\right)\right)-\pi\left(v_{-i}\right), 1\right)$ or $\left(\alpha_{0}\left(\pi\left(v_{-i}\right)\right), 0\right)$,

(ii) $v_{i}>\pi\left(v_{-i}\right)$ implies $i \in \varphi(v)$, and

(iii) $\pi\left(v_{-i}\right)>v_{i}$ implies $i \notin \varphi(v)$.

THEOREM 2: In the object setting, a rule $(\tau, \varphi)$ satisfies production efficiency, strategyproofness, and no-envy-in-trades if and only it is a citizen-shareholder rule.

The proof is in Appendix 7.

Remarkably, each citizen-shareholder rule can be viewed as a kind of equal-income Walrasian rule. In particular, consider a citizen-shareholder rule with social-dividend schedule $\alpha_{0}$, and let $v \in V$. Let $w$ denote the number of winners, and define

$$
\begin{aligned}
Y(v) & \equiv \alpha_{0}\left(\pi_{-}(v)\right), \\
p_{+}^{\text {Walras }}(v) & \equiv \pi_{+}(v)+\left(\alpha_{0}\left(\pi_{-}(v)\right)-\alpha_{0}\left(\pi_{+}(v)\right)\right), \text { and } \\
p_{-}^{\text {Walras }}(v) & \equiv 0 .
\end{aligned}
$$

Then the income $Y(v)$ and the price vector $\left(p_{+}^{\text {Walras }}(v), p_{-}^{\text {Walras }}(v)\right)$ together determine a budget set. By construction, each winner receives $Y(v)-p_{+}^{\text {Walras }}(v)$ and each loser receives $Y(v)-p_{-}^{\text {Walras }}(v)$. If $n>w>0$, then there is at least one winner and one loser, so by no-envy-in-trades, each winner selects his bundle from the budget set and each loser does as well. If $w=n$, then each agent wins and receives $\alpha_{0}\left(\mu_{n}\right)-\mu_{n}$, which he finds at least as desirable as losing and receiving $\alpha_{0}\left(\mu_{n}\right)$ as his value is at least $\mu_{n}$. If $w=0$, then each agent loses and receives $\alpha_{0}\left(\mu_{1}\right)$, which he finds at least as desirable as winning and receiving $\alpha_{0}\left(\mu_{1}\right)-\mu_{1}$ as his value is at most $\mu_{1}$. Thus in all cases, each agent receives a bundle he most-prefers from the common budget set.

Unsurprisingly, the price of an object is never negative; in fact it is always at least $\mu_{1}$. More surprisingly, the income $Y(v)$ can be positive, and in particular the total income received by the agents may exceed the amount of money initially in the economy. This is possible when the winners overpay; their overpayments can be used to finance the income for themselves and for the losers.

We now turn our attention to the constraints imposed by the no-deficit axiom.

Definition: A cost-constrained social-dividend schedule is a social-dividend schedule such that:

(i) $\alpha_{0}\left(\mu_{1}\right) \leq 0$,

(ii) for each $w \in\{1,2, \ldots, n-1\}$,

$$
(n-w) \alpha_{0}\left(\mu_{w+1}\right) \leq\left(w \mu_{w}-C(w)\right)-w \alpha_{0}\left(\mu_{w}\right), \text { and }
$$

(iii) $\alpha_{0}\left(\mu_{n}\right) \leq \frac{n \mu_{n}-C(n)}{n}$.

A rule $(\tau, \varphi)$ is a cost-constrained citizen-shareholder rule if (I) $(\tau, \varphi)$ is standard and (II) there is a cost-constrained social-dividend schedule $\alpha_{0}$ such that for each $i \in N$ and each $v_{-i} \in V_{-i},\left(\tau_{i}(v), \varphi_{i}(v)\right)$ is either $\left(\alpha_{0}\left(\pi\left(v_{-i}\right)\right)-\pi\left(v_{-i}\right), 1\right)$ or $\left(\alpha_{0}\left(\pi\left(v_{-i}\right)\right), 0\right)$.

Theorem 3: In the object setting, a rule $(\tau, \varphi)$ satisfies no-deficit, production efficiency, strategy-proofness and no-envy-in-trades if and only it is a cost-constrained citizenshareholder rule. 
The proof is in Appendix 8.

Finally, we turn our attention to (i) rules that are not Pareto-dominated, and (ii) our final axiom, voluntarism. First, we define the family of social-dividend schedules which most-aggressively increase to hit the social-dividend awards at the marginal costs:

Definition: A social-dividend schedule $\alpha_{0}$ is unrelenting if there is $\left(a_{w}\right) \in \mathbb{R}^{N}$ such that

(i) for each $w \in\{1,2, \ldots, n\}, \alpha_{0}\left(\mu_{w}\right)=a_{w}$, and

(ii) for each $w \in\{1,2, \ldots, n-1\}$ and each $\delta \in\left[0, \mu_{w+1}-\mu_{w}\right]$,

$$
\alpha_{0}\left(\mu_{w}+\delta\right)=\min \left\{\alpha_{0}\left(\mu_{w}\right)+\delta, \alpha_{0}\left(\mu_{w+1}\right)\right\} .
$$

In this case, we say that $\left(a_{w}\right)$ is the marginal cost award profile of $\alpha_{0}$.

As each unrelenting social-dividend schedule can be described by its marginal cost award profile, so too can the class of citizen-shareholder rules with such schedules that are not Pareto-dominated be described by its associated class of marginal cost award profiles. In particular, define the set of marginal cost award profiles $\mathcal{A} \subseteq \mathbb{R}^{N}$ by:

$$
\begin{aligned}
\mathcal{A} \equiv\left\{a \in \mathbb{R}^{N} \mid a_{1} \leq\right. & 0 ; \\
\text { for each } w \in\{1,2, \ldots, n-1\}, & \\
& a_{w+1}-a_{w} \leq \mu_{w+1}-\mu_{w}, \\
& a_{w+1}-a_{w} \geq 0, \text { and } \\
& (n-w) a_{w+1}+w a_{w} \leq w \mu_{w}-C(w) ; \text { and } \\
a_{n} \leq & \left.\frac{n \mu_{n}-C(n)}{n} .\right\}
\end{aligned}
$$

Define the subset of profiles that (we claim, and will show) are compatible with voluntary rules $\mathcal{A}^{V} \subseteq \mathcal{A}$ by:

$$
\mathcal{A}^{V} \equiv\left\{a \in \mathcal{A} \mid a_{1}=0\right\}
$$

To define the vector domination relation $\geq$, for each pair $a, a^{\prime} \in \mathbb{R}^{N}$, define $a \geq a^{\prime}$ if and only if for each $w \in\{1,2, \ldots, n\}, a_{w} \geq a_{w}^{\prime}$. If $a \geq a^{\prime}$ and $a \neq a^{\prime}$, we write $a>a^{\prime}$. Define the respective 'frontier' sets $\mathcal{F} \subseteq \mathcal{A}$ and $\mathcal{F}^{V} \subseteq \mathcal{A}^{V}$ by:

$$
\begin{aligned}
\mathcal{F} & \equiv\left\{a \in \mathcal{A} \mid a^{\prime} \in \mathcal{A} \text { and } a^{\prime} \geq a \text { implies } a^{\prime}=a\right\}, \text { and } \\
\mathcal{F}^{V} & \equiv\left\{a \in \mathcal{A}^{\mathcal{V}} \mid a^{\prime} \in \mathcal{A} \text { and } a^{\prime} \geq a \text { implies } a^{\prime}=a\right\} .
\end{aligned}
$$

THEOREM 4: In the object setting,

(I) a rule $(\tau, \varphi)$ (i) satisfies no-deficit, production efficiency, strategy-proofness, and no-envy-in-trades, and (ii) is not Pareto-dominated by another such rule, if and only if it is a cost-constrained citizen-shareholder rule with an unrelenting social-dividend schedule whose marginal cost award profile belongs to $\mathcal{F}$.

(II) a rule $(\tau, \varphi)$ (i) satisfies no-deficit, production efficiency, voluntarism, strategyproofness, and no-envy-in-trades, and (ii) is not Pareto-dominated by another such rule, if and only if it is a cost-constrained citizen-shareholder rule with an unrelenting socialdividend schedule whose marginal cost award profile belongs to $\mathcal{F}^{V}$.

The proof is in Appendix 9. 


\section{Conclusion}

We conclude by discussing several observations about our results.

\subsection{Asymmetric costs}

For our positive results, we imposed that the cost function is symmetric, but analogous results hold if costs are asymmetric in a structured way and no-envy-in-trades is adapted accordingly. In particular, suppose that-as before-production costs are given by a symmetric, finite, and convex function $C$. Now, however, in addition to these production costs, each agent $i \in N$ has a personal cost $\kappa_{i}$. For example, this might be the cost of shipping the object to agent $i$, or it might be the cost of further customizing the object to his specifications. Altogether, the cost of serving group $W$ is now given by:

$$
\hat{C}(W)=C(W)+\sum_{i \in W} \kappa_{i}
$$

In this setting, it is straightforward to modify our rules so that each agent necessarily pays his own personal cost should he win. In particular, for each $i \in N$, define $\hat{v}_{i} \equiv v_{i}+\kappa_{i}$. For each rule $(\tau, \varphi)$, define the rule $(\hat{\tau}, \hat{\varphi})$ by defining, for each $v \in V$ and each $i \in N$,

$$
\begin{aligned}
& \hat{\varphi}(\hat{v}) \equiv \varphi(v), \text { and } \\
& \hat{\tau}_{i}(\hat{v}) \equiv \tau_{i}(v)-\varphi_{i}(v) \kappa_{i} .
\end{aligned}
$$

For brevity, we use the past tense to refer to $(\tau, \varphi)$ and the present tense to refer to $(\hat{\tau}, \hat{\varphi})$. We claim that if $(\tau, \varphi)$ satisfied the axioms for $C$, then $(\hat{\tau}, \hat{\varphi})$ 'inherits' these axioms for $\hat{C}$ when no-envy-in-trades is properly adapted. Indeed, for each $v \in V$,

- since no-deficit was satisfied at $v$, it is now satisfied at $\hat{v}$ because each winner now covers his personal cost,

- since production efficiency was satisfied at $v$, it is now satisfied at $\hat{v}$ because each group has the same Marshallian surplus as before (since valuation increases are equal to cost increases), and

- since voluntarism was satisfied at $v$, it is now satisfied at $\hat{v}$ because a loser receives the same non-negative transfer, while a winner's valuation increases by the same amount as his contribution.

For strategy-proofness, it suffices to observe that for each $i \in N$, each $v \in V$, and each $v_{i}^{\prime} \in V_{i}$

$$
\begin{aligned}
v_{i} \varphi_{i}(v)+\tau_{i}(v) & =\hat{v}_{i} \hat{\varphi}_{i}(\hat{v})+\hat{\tau}_{i}(\hat{v}), \text { and } \\
v_{i} \varphi_{i}\left(v_{i}^{\prime}, v_{-i}\right)+\tau_{i}\left(v_{i}^{\prime}, v_{-i}\right) & =\hat{v}_{i} \hat{\varphi}_{i}\left(\hat{v}_{i}, \hat{v}_{-i}\right)+\hat{\tau}_{i}\left(\hat{v}_{i}, \hat{v}_{-i}\right) .
\end{aligned}
$$

from which the inheritance of strategy-proofness immediately follows.

To adapt no-envy-in-trades, we require that when an agent considers a peer's assignment, he first adjusts it by replacing his peer's personal cost with his own before comparing: 
No-ENVy-IN-ADJusted-TRAdes: For each pair $i, j \in N$ and each $v \in V$,

$$
v_{i} \varphi_{i}(v)+\tau_{i}(v) \geq v_{i} \varphi_{j}(v)+\tau_{j}(v)+\varphi_{j}(v)\left(\kappa_{j}-\kappa_{i}\right) .
$$

In other words, when $i$ considers the assignment of $j$, if $j$ wins, then in the adjustment $\kappa_{j}$ is returned and then $\kappa_{i}$ is deducted. It is straightforward to verify that if $(\tau, \varphi)$ satisfies no-envy-in-trades, then $(\hat{\tau}, \hat{\varphi})$ satisfies no-envy-in-adjusted-trades.

Altogether, then, in this special class of cost functions with asymmetric costs (which includes all additive cost functions), these natural analogues of our rules satisfy our requirements when the equal opportunity requirement is adjusted to take into account differences in personal costs.

\subsection{Two-sided economies}

Consider an economy with money and indivisible objects, where each agent desires at most one object and has preferences summarized by his valuation. Moreover, each agent belongs to one of two sides: each buyer is initially endowed with no object, while each seller is initially endowed with one object.

In such an economy, there are Walrasian price vectors (Koopmans and Beckmann, 1957; Gale, 1960), which have associated outcomes that form the core of a cooperative game for that economy (Shapley and Shubik, 1972). Moreover, the set of Walrasian price vectors is a bounded lattice (Shapley and Shubik, 1972), and when seller valuations are common knowledge, the minimum-price Walrasian rule, which uses reported buyer valuations to calculate the minimum Walrasian price vector and assign the associated core outcome, is strategy-proof (Demange, 1982; Leonard, 1983). ${ }^{11}$

Our analysis applies to the special case where the objects are identical. Indeed, though we originally presented $\mu_{1}, \mu_{2}, \ldots, \mu_{n}$ as marginal costs for the production technology, it would be just as legitimate to interpret these numbers as commonly-known seller valuations. In this special case, there is a single Walrasian price instead of a Walrasian price vector (Shapley and Shubik, 1972). Note that in this interpretation, a Walrasian outcome is Pareto-efficient: it does not involve burned money, but rather seller profit, and thus there is no violation of the First Welfare Theorem.

A rule in our class uses reported buyer valuations to calculate the minimum Walrasian price, but it does not necessarily recommend the associated core outcome, where each buyer receives his most-preferred bundle from the budget set given by this price and zero transfer. Instead, it may recommend an outcome where each buyer receives his mostpreferred bundle from the budget set given by this price and some positive transfer, which is an outcome outside of the core.

In the cooperative game for our Big Show example, the two fans who go to the show and the two scalpers who sell them tickets would together block our rule's recommendation. Thus it is only if the buyers can commit to honoring our rule's recommendation and not cooperating with the sellers that they might benefit, with respect to the Walrasian core outcome, at the sellers' expense. Similarly, in the market for horses considered by Böhm-Bawerk (1888), the horse-less might benefit over the classic solutions through such

\footnotetext{
${ }^{11}$ Demange and Gale (1985) showed that these facts persist even when preferences are not quasilinear-namely, that Walrasian prices form a lattice, and that the minimum-price Walrasian rule is strategy-proof - and this rule was recently characterized by Morimoto and Serizawa (2015).
} 
a pact. Whether such an agreement constitutes cooperation or collusion is a matter of perspective, varying from one side of the market to the other.

We remark that if, in our Big Show example, the fans are viewed as a nation that exports money, while the scalpers are viewed as a nation that exports tickets, then we have a (small) economy of international trade. For such economies, our rules suggest a role for a government that is dedicated only to the welfare of its own citizens.

\subsection{Markets versus central planning}

As notably emphasized by Hayek (1945), the solution to an economic problem typically depends on information that is scattered across different individuals, and historically, two institutions for reaching solutions have dominated economic analysis. In a market, a public summary of the scattered information is generated, and this summary shapes the opportunities available to individuals so that their independent choices are harmonious. In a (direct) mechanism, the scattered information is reported to a central authority, who calculates and administers a solution. While today, it is perhaps generally recognized that markets may be imperfect and mechanisms may help, historically (and especially during the Cold War), the issue was often painted in black and white. ${ }^{12}$ The relative merits of these institutions, cast as capitalism and socialism, were notably investigated during the socialist calculation debate beginning with von Mises (1920).

An important observation from that debate is that our (standard) definitions of markets and mechanisms do not preclude a hybrid institution (Lange 1936; Lerner 1944). Indeed, a market in Walrasian equilibrium summarizes information and shapes opportunities with a list of Walrasian (clearing) prices, which exist in general settings (Walras 1896; McKenzie 1954; Arrow and Debreu 1954), but it is not specified how this summary is generated; a mechanism calculates and administers a solution (Hurwicz 1972; Gibbard 1973), but it is not specified how this solution is administered. A Vickrey direct mechanism (Vickrey, 1961) fits both descriptions.

We believe that our analysis contributes to this debate. On the one hand, for a large class of industries, we find that centrally-planned production is necessarily unaffordable, inefficient, manipulable, or coercive. On the other hand, for a special class of industries, we find that central planning that uses market principles can in fact outperform the market, and to do so it need not resort to coercion. In such an institution, agents are incentivized to report their private information, then these reports are used to calculate an efficient production plan, and finally the agents voluntarily contribute the necessary funding in exchange for promised output. The central planner plays the role of the Walrasian auctioneer by calculating the Walrasian prices, but then goes further, calculating whether or not the agents over-contribute and returning social dividends to them when doing so does not interfere with incentives. Remarkably, precisely such an institution is described by Lange (1936):

It seems, therefore, convenient to regard the income of consumers as being composed of two parts: one part being the receipts for the labor services performed and the other part being a social dividend constituting the individual's

\footnotetext{
${ }^{12}$ For example, Milton Friedman (Friedman, 1962) writes: "Fundamentally, there are only two ways of co-ordinating the economic activities of millions. One is central direction involving the use of coercionthe technique of the army and of the modern totalitarian state. The other is voluntary co-operation of individuals - the technique of the market place."
} 
share in the income derived from the capital and the natural resources owned

by society.

This notion of a social dividend continues to play a role in models of market socialism (Bardhan and Roemer, 1992).

While our analysis suggests that for many industries, there would be some serious problems with such an institution, our analysis also suggests that for some very special industries, such an institution could help improve market outcomes - precisely by treating agents as citizen-shareholders and awarding social dividends. Theoretically, in such industries, a central computer could democratically and transparently guide production according to the interests of voluntary participants.

\section{Appendix 1}

In this appendix, we prove Theorem 1.

TheOREM 1: In the service setting, there is no rule satisfying no-deficit, production efficiency, voluntarism, and strategy-proofness.

Proof: Assume, by way of contradiction, $(\tau, \varphi)$ satisfies the given axioms. Define $\mathcal{W}_{f}$ to be the set of nonempty groups with finite cost:

$$
\mathcal{W}_{f} \equiv\left\{W \in 2^{N} \backslash\{\emptyset\} \mid C(W)<\infty\right\} .
$$

By weak returns, $\mathcal{W}_{f}$ is nonempty. We consider two cases.

CASE 1: For each $W \in \mathcal{W}_{f}$ and each $i \in W, C(\{i\})<\infty$.

For each $W \in \mathcal{W}_{f}$, define the group rate for $W, \Gamma(W)$, by:

$$
\Gamma(W) \equiv \frac{C(W)}{\sum_{W} C(\{i\})}
$$

By positive costs and the case's assumption, for each $W \in \mathcal{W}_{f}, \Gamma(W)$ is well-defined. Thus we can define the minimal group rate $\gamma$ by:

$$
\gamma \equiv \min _{W \in \mathcal{W}_{f}} \Gamma(W)
$$

By positive costs, $\gamma>0$, and by weak returns, $\gamma<1$.

Define the collection of groups with group rate $\gamma, \mathcal{W}_{\gamma}$, by:

$$
\mathcal{W}_{\gamma} \equiv\left\{W \in \mathcal{W}_{f} \mid \Gamma(W)=\gamma\right\}
$$

By construction, $\mathcal{W}_{\gamma}$ is nonempty. Let $W^{*}$ be an inclusion-minimal member of $\mathcal{W}_{\gamma}$ (that is, for each $\left.W \in \mathcal{W}_{\gamma}, W \not \subset W^{*}\right)$. Since $\gamma<1$, thus $\left|W^{*}\right| \geq 2$. By positive costs and the case's assumption, for each $i \in W^{*}, C(\{i\}) \in(0, \infty)$.

We proceed by first considering two successive profiles $v^{\prime}, v \in V$, then considering two families of profiles $\left\{v^{i^{\prime}}\right\}_{i \in W^{*}},\left\{v^{i}\right\}_{i \in W^{*}}$. First, define $v^{\prime} \in V$ by:

$$
v_{i}^{\prime}=\left\{\begin{array}{lr}
\gamma C(\{i\}), & i \in W^{*} \\
0, & \text { else. }
\end{array}\right.
$$


We claim $\emptyset$ and $W^{*}$ are the surplus-maximizers at $v^{\prime}$. Indeed:

- for $\emptyset, \sigma_{v^{\prime}}(\emptyset)=0$,

- for $W^{*}$,

$$
\begin{aligned}
\sigma_{v^{\prime}}\left(W^{*}\right) & =\sum_{W^{*}} \gamma C(\{i\})-C\left(W^{*}\right) \\
& =\gamma \sum_{W^{*}} C(\{i\})-C\left(W^{*}\right) \\
& =\frac{C\left(W^{*}\right)}{\sum_{W^{*}} C(\{i\})} \sum_{W^{*}} C(\{i\})-C\left(W^{*}\right) \\
& =0,
\end{aligned}
$$

- for each $W \in \mathcal{W}_{\gamma} \backslash\left\{W^{*}\right\}$, by construction there is $i^{\prime} \in W \backslash W^{*}$, and by positive costs and the case's assumption $C\left(\left\{i^{\prime}\right\}\right) \in(0, \infty)$, so

$$
\begin{aligned}
\sigma_{v^{\prime}}(W) & =\sum_{W \cap W^{*}} \gamma C(\{i\})-C(W) \\
& \leq \sum_{W \backslash\left\{i^{\prime}\right\}} \gamma C(\{i\})-C(W) \\
& <\sum_{W} \gamma C(\{i\})-C(W) \\
& =\gamma \sum_{W} C(\{i\})-C(W) \\
& \leq \frac{C(W)}{\sum_{W} C(\{i\})} \sum_{W} C(\{i\})-C(W) \\
& =0
\end{aligned}
$$

- for each $W \in \mathcal{W}_{f} \backslash \mathcal{W}_{\gamma}$,

$$
\begin{aligned}
\sigma_{v^{\prime}}(W) & =\sum_{W \cap W^{*}} \gamma C(\{i\})-C(W) \\
& \leq \sum_{W} \gamma C(\{i\})-\frac{C(W)}{\sum_{W} C(\{i\})} \sum_{W} C(\{i\} \\
& =\left(\gamma-\frac{C(W)}{\sum_{W} C(\{i\})}\right) \sum_{W} C(\{i\}) \\
& <0, \text { and }
\end{aligned}
$$

- for each $W \in 2^{N} \backslash \mathcal{W}_{f}, \sigma_{v^{\prime}}(W)=-\infty$.

Thus there is $\epsilon \in(0,1)$ such that

(i) for each $W \in 2^{N} \backslash\left\{\emptyset, W^{*}\right\}, \sigma_{v^{\prime}}\left(W^{*}\right)-\epsilon>\sigma_{v^{\prime}}(W)$, and

(ii) for each $i \in W^{*}, v_{i}^{\prime}-\epsilon \geq 0$.

Define $v \in V$ by:

$$
v_{i}=\left\{\begin{array}{lr}
\gamma C(\{i\})+1, & i \in W^{*} \\
0, & \text { else. }
\end{array}\right.
$$


We claim $W^{*}$ is the unique surplus-maximizer at $v$. Indeed, each group's surplus increases by at most $\left|W^{*}\right|$ from $v^{\prime}$ to $v$, and the surplus of $W^{*}$ increases by this amount while the surplus of $\emptyset$ does not. Thus by production efficiency, $\varphi(v)=W^{*}$.

For each $i \in W^{*}$, define $v^{i^{\prime}}, v^{i} \in V$ by:

$$
\begin{aligned}
& v_{j}^{i^{\prime}}= \begin{cases}v_{i}^{\prime}-\epsilon, & j=i, \\
v_{j}^{\prime}, & \text { else. }\end{cases} \\
& v_{j}^{i}= \begin{cases}v_{i}^{\prime}-\epsilon, & j=i, \\
v_{j}, & \text { else. }\end{cases}
\end{aligned}
$$

By construction of $\epsilon$, these are indeed profiles in $V$. Let $i \in W^{*}$. We claim $W^{*}$ is the unique surplus-maximizer at $v^{i}$. Indeed:

- for $\emptyset$, since $\left|W^{*}\right| \geq 2$ and by construction of $\epsilon$,

$$
\begin{aligned}
\sigma_{v^{i}}\left(W^{*}\right) & =\sigma_{v^{i^{\prime}}}\left(W^{*}\right)+\left(\left|W^{*}\right|-1\right) \\
& \geq \sigma_{v^{i^{\prime}}}\left(W^{*}\right)+1 \\
& =\left(\sigma_{v^{\prime}}\left(W^{*}\right)-\epsilon\right)+1 \\
& >\sigma_{v^{\prime}}\left(W^{*}\right) \\
& =0 \\
& =\sigma_{v^{i}}(\emptyset), \text { and }
\end{aligned}
$$

- for $W \in 2^{N} \backslash\left\{\emptyset, W^{*}\right\}$, by construction of $\epsilon$,

$$
\begin{aligned}
\sigma_{v^{i^{\prime}}}\left(W^{*}\right) & =\sigma_{v^{\prime}}\left(W^{*}\right)-\epsilon \\
& >\sigma_{v^{\prime}}(W) \\
& \geq \sigma_{v^{i^{\prime}}}(W),
\end{aligned}
$$

so since only members of $W^{*}$ have higher valuations at $v^{i}$ than $v^{i^{\prime}}$, thus $\sigma_{v^{i}}\left(W^{*}\right)>\sigma_{v^{i}}(W)$.

By production efficiency, $\varphi\left(v^{i}\right)=W^{*}$, so by voluntarism, $\tau_{i}\left(v^{i}\right) \geq-v_{i}^{i}>-\gamma C(\{i\})$, so by strategy-proofness, $\tau_{i}(v) \geq \tau_{i}\left(v^{i}\right)>-\gamma C(\{i\})$.

Since $i \in W^{*}$ was arbitrary, thus for each $i \in W^{*}, \tau_{i}(v)>-\gamma C(\{i\})$. By voluntarism, for each $i \in N \backslash W^{*}, \tau_{i}(v) \geq 0$. But then

$$
\begin{aligned}
\sum \tau_{i}(v) & >-\gamma \sum_{W^{*}} C(\{i\}) \\
& =-C\left(W^{*}\right) \\
& =-C(\varphi(v)),
\end{aligned}
$$

contradicting no-deficit.

CAsE 2: There is $W \in \mathcal{W}_{f}$ and $i \in W$ such that $C(\{i\})=\infty$.

Define $M$ to be the maximum finite cost:

$$
M \equiv \max _{\mathcal{W}_{f}} C(W)
$$


For each $i \in \cup \mathcal{W}_{f}$ such that $C(\{i\})=\infty$, define $C^{*}(\{i\}) \equiv M+1$. For all other groups, define $C^{*}(W) \equiv C(W)$. It is easy to verify that $C^{*}$ satisfies weak returns and positive costs, and thus satisfies the Case 1 assumptions.

For each axiom, let us distinguish the version involving $C$ and the version involving $C^{*}$ by writing $C$-axiom and $C^{*}$-axiom; for example, $C$-no-deficit and $C^{*}$-no-deficit. Similarly, we write $C$-surplus and $C^{*}$-surplus.

By assumption, $(\tau, \varphi)$ satisfies the $C$-axioms. We claim that furthermore, $(\tau, \varphi)$ satisfies the $C^{*}$-axioms. Indeed, both versions of strategy-proofness and voluntarism are equivalent, as neither axiom makes reference to the cost function. Moreover, $C^{*}$-nodeficit cannot be violated because no group has a higher cost under $C^{*}$ than under $C$. Finally, all groups have the same $C$-surplus and $C^{*}$-surplus except the singletons from $\cup \mathcal{W}_{f}$, so a violation of $C^{*}$-production efficiency must involve a valuation profile $v$ where such a singleton $\{i\}$ has the highest $C^{*}$-surplus, but is not selected. But there is $W \in \mathcal{W}_{f}$ such that $i \in W$, and by construction the $C^{*}$-surplus of $W$ is higher than the $C^{*}$-surplus of $i$ at any profile, contradicting that $\{i\}$ has the highest $C^{*}$-surplus at $v$.

Altogether, $(\tau, \varphi)$ satisfies the $C^{*}$-axioms, contradicting that there is no such rule by Case 1.

\section{Appendix 2}

In this appendix, we prove the Bidding Lemma.

BIDDING LEMMA: In the object setting, a rule $(\tau, \varphi)$ satisfies production efficiency if and only if for each $v \in V$ with $w \equiv|\varphi(v)|$,

(i) for each $i \in \varphi(v)$ and each $j \in N \backslash \varphi(v), v_{i} \geq v_{j}$,

(ii) $w \neq 0$ implies $B_{w}(v) \geq \mu_{w}$, and

(iii) $w \neq n$ implies $\mu_{w+1} \geq B_{w+1}(v)$.

Proof: We establish both implications.

$[\Rightarrow]$ Let $(\tau, \varphi)$ satisfy production efficiency, let $v \in V$, and define $w \equiv|\varphi(v)|$.

(i) Assume, by way of contradiction, there are $i \in \varphi(v)$ and $j \in N \backslash \varphi(v)$ such that $v_{j}>v_{i}$. Then

$$
\begin{aligned}
\sigma_{v}((\varphi(v) \backslash\{i\}) \cup\{j\}) & =\sum_{(\varphi(v) \backslash\{i\}) \cup\{j\}} v_{k}-C(|(\varphi(v) \backslash\{i\}) \cup\{j\}|) \\
& =\sum_{\varphi(v)} v_{k}+\left(v_{j}-v_{i}\right)-C(|\varphi(v)|) \\
& >\sum_{\varphi(v)} v_{k}-C(|\varphi(v)|) \\
& =\sigma_{v}(\varphi(v)),
\end{aligned}
$$

contradicting that $\varphi$ satisfies production efficiency.

(ii) Assume, by way of contradiction, $w \neq 0$ and $\mu_{w}>B_{w}(v)$. By (i), there is $i \in \varphi(v)$ 
with $v_{i}=B_{w}(v)$. But then

$$
\begin{aligned}
\sigma_{v}(\varphi(v) \backslash\{i\}) & =\sum_{\varphi(v) \backslash\{i\}} v_{k}-C(|\varphi(v) \backslash\{i\}|) \\
& =\sum_{\varphi(v)} v_{k}-B_{w}(v)-\left(C(w)-\mu_{w}\right) \\
& =\sigma_{v}(\varphi(v))+\left(\mu_{w}-B_{w}(v)\right) \\
& >\sigma_{v}(\varphi(v)),
\end{aligned}
$$

contradicting that $\varphi$ satisfies production efficiency.

(iii) Assume, by way of contradiction, $w \neq n$ and $B_{w+1}(v)>\mu_{w+1}$. By (i), there is $i \in N \backslash \varphi(v)$ with $v_{i}=B_{w+1}(v)$. But then

$$
\begin{aligned}
\sigma_{v}(\varphi(v) \cup\{i\}) & =\sum_{\varphi(v) \cup\{i\}} v_{k}-C(|\varphi(v) \cup\{i\}|) \\
& =\sum_{\varphi(v)} v_{k}+B_{w+1}(v)-\left(C(w)+\mu_{w+1}\right) \\
& =\sigma_{v}(\varphi(v))+\left(B_{w+1}(v)-\mu_{w+1}\right) \\
& >\sigma_{v}(\varphi(v)),
\end{aligned}
$$

contradicting that $\varphi$ satisfies production efficiency.

$[\Leftarrow]$ Assume, by way of contradiction, $(\tau, \varphi)$ satisfies the hypotheses but does not satisfy production efficiency. Then there are $v \in V$ and $W \subseteq N$ such that $\sigma_{v}(W)>\sigma_{v}(\varphi(v))$. Let $W^{*} \subseteq N$ be a group of $|W|$ agents such that $i \in W^{*}$ and $v_{j}>v_{i}$ implies $j \in W^{*}$. Since $C\left(W^{*}\right)=C(W)$, thus $\sigma_{v}\left(W^{*}\right) \geq \sigma_{v}(W)>\sigma_{v}(\varphi(v))$.

Let $\sigma^{*}(w)$ denote the surplus at $v$ for serving $w$ highest-valuation agents. Formally, define $\sigma^{*}:\{0,1, \ldots, n\} \rightarrow \mathbb{R}$ by: $\sigma^{*}(0)=0$, and for each $w \in\{1,2, \ldots, n\}$,

$$
\sigma^{*}(w)=\sum_{r=1}^{w}\left(B_{r}(v)-\mu_{r}\right) .
$$

By construction of $W^{*}$ and by (i), $\sigma^{*}\left(\left|W^{*}\right|\right)=\sigma_{v}\left(W^{*}\right)>\sigma_{v}(\varphi(v))=\sigma^{*}(|\varphi(v)|)$.

For each $w \in\{1,2, \ldots, n\}$, since $B_{w}(v)$ is non-increasing in $w$ and $\mu_{w}$ is non-decreasing in $w$, thus $B_{w}(v)-\mu_{w}$ is non-increasing in $w$. Then $\sigma^{*}$ is maximized at $w$ if $\sigma^{*}(w)$ sums across all positive terms and no negative terms, or if:

- $w \neq 0$ implies $B_{w}(v)-\mu_{w} \geq 0$ and

- $w \neq n$ implies $0 \geq B_{w+1}(v)-\mu_{w+1}$.

But by (i), (ii), and (iii), these conditions are met for $|\varphi(v)|$, so $\sigma^{*}$ is maximized at $|\varphi(v)|$, contradicting $\sigma^{*}\left(\left|W^{*}\right|\right)>\sigma^{*}(|\varphi(v)|)$.

\section{Appendix 3}

In this appendix, we prove the Price Lemma.

PRICE Lemma: In the object setting, a rule $(\tau, \varphi)$ satisfies strategy-proofness if and only if for each $i \in N$ and each $v_{-i} \in V_{-i}$, there are $\tau_{i}^{+}\left(v_{-i}\right), \tau_{i}^{-}\left(v_{-i}\right) \in \mathbb{R}$ and $p_{i}\left(v_{-i}\right) \in$ $\mathbb{R} \cup\{-\infty, \infty\}$ such that for each $v_{i} \in V_{i}$, 
(i) $\left(\tau_{i}(v), \varphi_{i}(v)\right)$ is either $\left(\tau_{i}^{+}\left(v_{-i}\right), 1\right)$ or $\left(\tau_{i}^{-}\left(v_{-i}\right), 0\right)$,

(ii) $p_{i}\left(v_{-i}\right) \notin\{-\infty, \infty\}$ implies $\tau_{i}^{+}\left(v_{-i}\right)=\tau_{i}^{-}\left(v_{-i}\right)-p_{i}\left(v_{-i}\right)$,

(iii) $v_{i}>p_{i}\left(v_{-i}\right)$ implies $i \in \varphi(v)$, and

(iv) $p_{i}\left(v_{-i}\right)>v_{i}$ implies $i \notin \varphi(v)$.

Proof: We establish both implications.

$[\Rightarrow]$ Let $(\tau, \varphi)$ satisfy strategy-proofness, let $i \in N$, and let $v_{-i} \in V_{-i}$. We begin by defining $\tau_{i}^{+}\left(v_{-i}\right), \tau_{i}^{-}\left(v_{-i}\right)$, and $p_{i}\left(v_{-i}\right)$.

If there are $v_{i}, v_{i}^{\prime} \in V_{i}$ such that $i \in \varphi(v)$ and $i \in \varphi\left(v_{i}^{\prime}, v_{-i}\right)$, then by strategy-proofness $\tau_{i}(v)=\tau_{i}\left(v_{i}^{\prime}, v_{-i}\right)$. Thus if there is $v_{i}^{+} \in V_{i}$ such that $i \in \varphi\left(v_{i}^{+}, v_{-i}\right)$, then there is a unique $\tau_{i}^{+}\left(v_{-i}\right) \in \mathbb{R}$ such that for each $v_{i} \in V_{i}, i \in \varphi(v)$ implies $\tau_{i}(v)=\tau_{i}^{+}\left(v_{-i}\right)$. If there is no such $v_{i}^{+} \in V_{i}$, let $\tau_{i}^{+}\left(v_{-i}\right) \in \mathbb{R}$ be arbitrary.

Similarly, if there is $v_{i}^{-} \in V_{i}$ such that $i \notin \varphi\left(v_{i}^{-}, v_{-i}\right)$, then there is a unique $\tau_{i}^{-}\left(v_{-i}\right) \in$ $\mathbb{R}$ such that for each $v_{i} \in V_{i}, i \notin \varphi(v)$ implies $\tau_{i}(v)=\tau_{i}^{-}\left(v_{-i}\right)$; if there is no such $v_{i}^{-} \in V_{i}$, let $\tau_{i}^{-}\left(v_{-i}\right) \in \mathbb{R}$ be arbitrary.

If there are $v_{i}^{+}, v_{i}^{-} \in V_{i}$ such that $i \in \varphi\left(v_{i}^{+}, v_{-i}\right)$ and $i \notin \varphi\left(v_{i}^{-}, v_{-i}\right)$, then define $p_{i}\left(v_{-i}\right) \equiv \tau_{i}^{-}\left(v_{-i}\right)-\tau_{i}^{+}\left(v_{-i}\right)$. If for each $v_{i} \in V_{i}, i \in \varphi(v)$, then define $p_{i}\left(v_{-i}\right) \equiv-\infty$. If for each $v_{i} \in V_{i}, i \notin \varphi(v)$, then define $p_{i}\left(v_{-i}\right) \equiv \infty$.

By construction, (i) and (ii) hold. If $p_{i}\left(v_{-i}\right) \in\{-\infty, \infty\}$, then it is immediate that (iii) and (iv) hold as well, so assume $p_{i}\left(v_{-i}\right) \in \mathbb{R}$. Then there are $v_{i}^{+}, v_{i}^{-} \in V_{i}$ such that $i \in \varphi\left(v_{i}^{+}, v_{-i}\right)$ and $i \notin \varphi\left(v_{i}^{-}, v_{-i}\right)$. Moreover, $p_{i}\left(v_{-i}\right)=\tau_{i}^{-}\left(v_{-i}\right)-\tau_{i}^{+}\left(v_{-i}\right)$, so with valuation $p_{i}\left(v_{-i}\right) \in V_{i}, i$ is indifferent between $\left(\tau_{i}^{+}\left(v_{-i}\right), 1\right)$ and $\left(\tau_{i}^{-}\left(v_{-i}\right), 0\right)$. If $v_{i}>p_{i}\left(v_{-i}\right)$, then $\left(\tau_{i}^{+}\left(v_{-i}\right), 1\right)$ is preferred and is attainable by reporting $v_{i}^{+}$, so by strategy-proofness it must be attained by reporting $v_{i}$, so $i \in \varphi(v)$. Similarly, $p_{i}\left(v_{-i}\right)>v_{i}$ implies $i \notin \varphi(v)$; thus (iii) and (iv) hold, as desired.

$[\Leftarrow]$ Assume $(\tau, \varphi)$ satisfies the hypotheses. Let $i \in N, v \in V$, and $v_{i}^{\prime} \in V_{i}$. If $p_{i}\left(v_{-i}\right) \in$ $\{-\infty, \infty\}$, then by (iii) and (iv) $\left(\tau_{i}(v), \varphi_{i}(v)\right)=\left(\tau_{i}\left(v_{i}^{\prime}, v_{-i}\right), \varphi_{i}\left(v_{i}^{\prime}, v_{-i}\right)\right)$ and we are done. If not, by (ii) $p_{i}\left(v_{-i}\right)=\tau_{i}^{-}\left(v_{-i}\right)-\tau_{i}^{+}\left(v_{-i}\right)$. If $\varphi_{i}(v)=\varphi_{i}\left(v_{i}^{\prime}, v_{-i}\right)$, then by (i) $\left(\tau_{i}(v), \varphi_{i}(v)\right)=$ $\left(\tau_{i}\left(v_{i}^{\prime}, v_{-i}\right), \varphi_{i}\left(v_{i}^{\prime}, v_{-i}\right)\right)$ and we are done, so assume either $i \in \varphi(v) \backslash \varphi\left(v_{i}^{\prime}, v_{-i}\right)$ or $i \in$ $\varphi\left(v_{i}^{\prime}, v_{-i}\right) \backslash \varphi(v)$.

If $i \in \varphi(v) \backslash \varphi\left(v_{i}^{\prime}, v_{-i}\right)$, then by (iv) $v_{i} \geq p_{i}\left(v_{-i}\right)$, so by (i)

$$
\begin{aligned}
v_{i} \varphi_{i}(v)+\tau_{i}(v) & \geq p_{i}\left(v_{-i}\right)+\tau_{i}^{+}\left(v_{-i}\right) \\
& =\left(\tau_{i}^{-}\left(v_{-i}\right)-\tau_{i}^{+}\left(v_{-i}\right)\right)+\tau_{i}^{+}\left(v_{-i}\right) \\
& =\tau_{i}^{-}\left(v_{-i}\right) \\
& =v_{i} \varphi_{i}\left(v_{i}^{\prime}, v_{-i}\right)+\tau_{i}\left(v_{i}^{\prime}, v_{-i}\right)
\end{aligned}
$$

as desired. If $i \in \varphi\left(v_{i}^{\prime}, v_{-i}\right) \backslash \varphi(v)$, then by (iii) $p_{i}\left(v_{-i}\right) \geq v_{i}$, so by (i)

$$
\begin{aligned}
v_{i} \varphi_{i}(v)+\tau_{i}(v) & =\tau_{i}^{-}\left(v_{-i}\right) \\
& =\left(\tau_{i}^{-}\left(v_{-i}\right)-\tau_{i}^{+}\left(v_{-i}\right)\right)+\tau_{i}^{+}\left(v_{-i}\right) \\
& =p_{i}\left(v_{-i}\right)+\tau_{i}^{+}\left(v_{-i}\right) \\
& \geq v_{i} \varphi_{i}\left(v_{i}^{\prime}, v_{-i}\right)+\tau_{i}\left(v_{i}^{\prime}, v_{-i}\right)
\end{aligned}
$$

as desired. 


\section{Appendix 4}

In this appendix, we prove the Auction Lemma.

Auction Lemma: In the object setting, a rule $(\tau, \varphi)$ satisfies production efficiency and strategy-proofness if and only if it is a peer-consolation rule.

Proof: We establish both implications.

$[\Rightarrow]$ Let $(\tau, \varphi)$ be a rule satisfying production efficiency and strategy-proofness. By the Price Lemma, there are $\tau_{i}^{+}\left(v_{-i}\right), \tau_{i}^{-}\left(v_{-i}\right) \in \mathbb{R}$ and $p_{i}\left(v_{-i}\right) \in \mathbb{R}$ satisfying the stated requirements. ${ }^{13}$

Let $i \in N$ and let $v_{-i} \in V_{-i}$. Define $\pi \equiv \pi\left(v_{-i}\right)$ and define $w \equiv \Omega\left(v_{-i}\right)$. By definition, $B_{w}\left(v_{-i}\right) \geq \mu_{w}$ and, if $w<n-1, \mu_{w+1}>B_{w+1}\left(v_{-i}\right)$. We claim that $v_{i}>\pi$ implies $i \in \varphi(v)$ and $\pi>v_{i}$ implies $i \notin \varphi(v)$.

CASE 1: $w>0$ and $v_{i}>B_{w}\left(v_{-i}\right)$. If $w>|\varphi(v)|$, then by the definition of $w$ and by the Bidding Lemma,

$$
\begin{aligned}
v_{i} & >B_{w}\left(v_{-i}\right) \\
& \geq \mu_{w} \\
& \geq \mu_{|\varphi(v)|+1} \\
& \geq B_{|\varphi(v)|+1}(v) .
\end{aligned}
$$

Thus $i$ is in any group of $|\varphi(v)|$ highest bidders, so by the Bidding Lemma, $i \in \varphi(v)$.

If $|\varphi(v)| \geq w$, then since $i$ is in any group of $w$ highest bidders, thus $i$ is in any group of $|\varphi(v)|$ highest bidders, so by the Bidding Lemma $i \in \varphi(v)$.

CASE 2: $v_{i}>\mu_{w+1}$. If $w=n-1$, then by production efficiency $i \in \varphi(v)$, so assume $n-1>w$.

- If $w=0$, then $v_{i}>\mu_{1}>B_{1}\left(v_{-i}\right)$, so by production efficiency $\varphi(v)=\{i\}$.

- If $w>0$ and $v_{i}>B_{w}\left(v_{-i}\right)$, then by Case $1, i \in \varphi(v)$.

- Assume $w>0$ and $B_{w}\left(v_{-i}\right) \geq v_{i}$. Then $B_{w}\left(v_{-i}\right) \geq v_{i}>\mu_{w+1}>B_{w+1}\left(v_{-i}\right)$, so $B_{w+1}(v)=v_{i}>\mu_{w+1}$ and $\mu_{w+2} \geq \mu_{w+1}>B_{w+1}\left(v_{-i}\right)=B_{w+2}(v)$. Thus by the Bidding Lemma, $|\varphi(v)|=w+1$ and $i \in \varphi(v)$.

CASE 3: $\pi>v_{i}$. Assume, by way of contradiction, $i \in \varphi(v)$.

If $w=0$, then $\mu_{1}>v_{i}$, so $\sigma_{v}(\varphi(v) \backslash\{i\})>\sigma_{v}(\varphi(v))$, contradicting that $\varphi$ satisfies production efficiency.

Assume $w>0$. By the Bidding Lemma, $i$ is one of the $|\varphi(v)|$ highest bidders, so as $B_{w}\left(v_{-i}\right)>v_{i}$, thus $|\varphi(v)|>w$. But then $|\varphi(v)| \geq w+1$, so by the Bidding Lemma, $v_{i} \geq \mu_{|\varphi(v)|} \geq \mu_{w+1}$, contradicting $\mu_{w+1}>v_{i}$.

In each case our claim is true, so $p_{i}\left(v_{-i}\right)=\pi=\pi\left(v_{-i}\right)$. Define $\alpha_{i}\left(v_{-i}\right) \equiv \tau_{i}^{-}\left(v_{-i}\right)$ and by the Price Lemma we are done.

\footnotetext{
${ }^{13}$ Using production efficiency, we can rule out $p_{i}\left(v_{-i}\right) \in\{-\infty, \infty\}$ : since $0 \leq \mu_{1}<\infty$, we can always find $v_{i}^{+}$so that $i$ wins and $v_{i}^{-}$so that $i$ loses.
} 
$[\Leftarrow]$ Assume that $(\tau, \varphi)$ satisfies the requirements and let $\left(\alpha_{i}\right)$ be its list of peer-consolation schedules. Since $(\tau, \varphi)$ is a peer-consolation rule, it satisfies production efficiency. For each $i \in N$ and each $v_{-i} \in V_{-i}$, define $\tau_{i}^{+}\left(v_{-i}\right) \equiv \alpha_{i}\left(v_{-i}\right)-\pi\left(v_{-i}\right), \tau_{i}^{-}\left(v_{-i}\right) \equiv \alpha_{i}\left(v_{-i}\right)$, and $p_{i}\left(v_{-i}\right) \equiv \pi\left(v_{-i}\right)$. It is straightforward to verify that these satisfy the requirements of the Price Lemma, so $(\tau, \varphi)$ is strategy-proof.

\section{Appendix 5}

In this appendix, we prove the Winner-Loser Prices Lemma.

Winner-Loser Prices Lemma: In the object setting, for each $v \in V$ and each $i \in N$,

(i) $v_{i} \geq \pi\left(v_{-i}\right)$ implies $\pi\left(v_{-i}\right)=\pi_{+}(v)$, and

(ii) $\pi\left(v_{-i}\right) \geq v_{i}$ implies $\pi\left(v_{-i}\right)=\pi_{-}(v)$.

Moreover, either $v_{i} \geq \pi_{-}(v) \geq \pi_{+}(v)$ or $\pi_{-}(v) \geq \pi_{+}(v) \geq v_{i}$.

Proof: Let $v \in V$ and let $i \in N$. Define $\pi_{i} \equiv \pi\left(v_{-i}\right)$, define $w \equiv \Omega(v)$, and define $w_{i} \equiv \Omega\left(v_{-i}\right)$. By definition, in $N$ at least $w$ agents bid at least $\mu_{w}$ and (if $n>w$ ) less than $w+1$ agents bid at least $\mu_{w+1}$. Similarly, in $N \backslash\{i\}$ at least $w_{i}$ agents bid at least $\mu_{w_{i}}$ and less than $w_{i}+1$ agents bid at least $\mu_{w_{i}+1}$.

If $w=n$, then $w_{i}=n-1$ and $B_{n-1}\left(v_{-i}\right) \geq \mu_{n}$, so $\pi_{i}=\mu_{n}$. If $w=0$, then $w_{i}=0$, so $\pi_{i}=\mu_{1}$. Assume $n>w>0$.

CASE 1: $\mu_{w_{i}+1}>B_{w_{i}}\left(v_{-i}\right)$. Then $\pi_{i}=B_{w_{i}}\left(v_{-i}\right)$, and in $N \backslash\{i\}$ at least $w_{i}$ agents bid at least $\pi_{i}$ and less than $w_{i}$ agents bid more than $\pi_{i}$. Thus in $N$, regardless of what $i$ bids, at least $w_{i}$ agents bid at least $\pi_{i}$, which is at least $\mu_{w_{i}}$, and less than $w_{i}+1$ agents bid more than $\pi_{i}$, which is at most $\mu_{w_{i}+1}$, so $w=w_{i}$.

If $v_{i} \geq \pi_{i}$, then $v_{i} \geq B_{w}\left(v_{-i}\right)$, so $B_{w+1}(v)=B_{w}\left(v_{-i}\right)=\pi_{i}$. Since $B_{w+1}(v)=$ $B_{w_{i}}\left(v_{-i}\right) \geq \mu_{w_{i}}=\mu_{w}$, altogether $\pi_{i}=\max \left\{B_{w+1}(v), \mu_{w}\right\}$, as desired.

If $\pi_{i} \geq v_{i}$, then $B_{w}\left(v_{-i}\right) \geq v_{i}$, so $B_{w}(v)=B_{w}\left(v_{-i}\right)=\pi_{i}$. Since $\mu_{w+1}>B_{w_{i}}\left(v_{-i}\right)=$ $B_{w}(v)$, altogether $\pi_{i}=\min \left\{B_{w}(v), \mu_{w+1}\right\}$, as desired.

CASE 2: $B_{w_{i}}\left(v_{-i}\right) \geq \mu_{w_{i}+1}$. Then $\pi_{i}=\mu_{w_{i}+1}$, and in $N \backslash\{i\}$ exactly $w_{i}$ agents bid at least $\pi$.

If $v_{i} \geq \pi_{i}$, then in $N$ exactly $w_{i}+1$ agents bid at least $\pi_{i}=\mu_{w_{i}+1}$, so $w=w_{i}+1$ and $\pi_{i}=\mu_{w}$. Since $v_{i} \geq \pi_{i}=\mu_{w_{i}+1}>B_{w_{i}+1}\left(v_{-i}\right)$, thus $B_{w_{i}+1}\left(v_{-i}\right)=B_{w_{i}+2}(v)=$ $B_{w+1}(v)$. Since $\mu_{w}=\mu_{w_{i}+1}>B_{w_{i}+1}\left(v_{-i}\right)=B_{w+1}(v)$, altogether $\pi_{i}=\max \left\{B_{w+1}(v), \mu_{w}\right\}$, as desired.

In the special case that $v_{i}=\pi_{i}=\mu_{w}$, we have that $v_{i}=B_{w_{i}+1}(v)=B_{w}(v)$. Since $\mu_{w+1} \geq \mu_{w}=v_{i}=B_{w}(v)$, thus $\pi_{i}=\min \left\{B_{w}(v), \mu_{w+1}\right\}$, as desired.

If $\pi>v_{i}$, then in $N$ exactly $w_{i}$ agents bid at least $\pi_{i}=\mu_{w_{i}+1}$, so $w=w_{i}$ and $\pi_{i}=\mu_{w+1}$. Since $B_{w_{i}}\left(v_{-i}\right) \geq \mu_{w_{i}+1}=\pi_{i}>v_{i}$, thus $B_{w_{i}}\left(v_{-i}\right)=B_{w_{i}}(v)=B_{w}(v)$. Since $B_{w}(v)=B_{w_{i}}\left(v_{-i}\right) \geq \mu_{w_{i}+1}=\mu_{w+1}$, altogether $\pi_{i}=\min \left\{B_{w}(v), \mu_{w+1}\right\}$, as desired.

Conclusion: If $w \in\{0, n\}$, then $\pi_{-}(v)=\pi_{+}(v)$. If $n>w>0$, then $B_{w}(v) \geq$ $B_{w+1}(v), B_{w}(v) \geq \mu_{w}, \mu_{w+1}>B_{w+1}$, and $\mu_{w+1} \geq \mu_{w}$, so altogether $\pi_{-}(v) \geq \pi_{+}(v)$.

Assume, by way of contradiction, $\pi_{-}(v)>v_{i}>\pi_{+}(v)$. Then $n>w>0$ and $B_{w}(v)>v_{i}>B_{w+1}(v)$, which is impossible. 


\section{Appendix 6}

In this appendix, we prove the Consolation Lemma.

Consolation Lemma: In the object setting, if a rule $(\tau, \varphi)$ satisfies production efficiency, strategy-proofness, and no-envy-in-trades, then $(\tau, \varphi)$ is a peer-consolation rule with peer-consolation schedules $\left(\alpha_{i}\right)$ such that for each $i \in N$ and each pair $v_{-i}, v_{-i}^{\prime} \in V_{-i}$,

$$
\pi\left(v_{-i}\right)=\pi\left(v_{-i}^{\prime}\right) \text { implies } \alpha_{i}\left(v_{-i}\right)=\alpha_{i}\left(v_{-i}^{\prime}\right) .
$$

Proof: Assume that $(\tau, \varphi)$ satisfies production efficiency, strategy-proofness and noenvy-in-trades. By the Auction Lemma, $(\tau, \varphi)$ is a peer-consolation rule. Let $\left(\alpha_{i}\right)$ be its peer-consolation schedules. By no-envy-in-trades, for each $v \in V$, there are $\tau_{+}(v), \tau_{-}(v) \in$ $\mathbb{R}$ such that $i \in \varphi(v)$ implies $\tau_{i}(v)=\tau_{+}(v)$ and $i \notin \varphi(v)$ implies $\tau_{i}(v)=\tau_{-}(v)$.

Let $i \in N$ and let $\pi \in\left[\mu_{1}, \mu_{n}\right]$. We claim that for each pair $v_{-i}, v_{-i}^{\prime} \in V_{-i}$ such that $\pi\left(v_{-i}\right)=\pi\left(v_{-i}^{\prime}\right)=\pi, \alpha_{i}\left(v_{-i}\right)=\alpha_{i}\left(v_{-i}^{\prime}\right)$.

CASE $1:^{14} \pi \in\left[\mu_{1}, \mu_{n}\right] \backslash\left\{\mu_{1}, \mu_{2}, \ldots, \mu_{n}\right\}$. Let $v_{-i} \in V_{-i}$ with $\pi\left(v_{-i}\right)=\pi$, and define $w \equiv \Omega\left(v_{-i}\right)$. By construction, $B_{w}\left(v_{-i}\right)=\pi, \mu_{w+1} \geq B_{w}\left(v_{-i}\right)$, and $B_{w}\left(v_{-i}\right) \geq \mu_{w}$. Since $\pi \notin\left\{\mu_{w}, \mu_{w+1}\right\}$, thus $\pi \in\left(\mu_{w}, \mu_{w+1}\right)$. Define $v^{*} \in V$ to be the profile where each agent reports $\pi$.

Define $v_{i} \equiv \max \left\{B_{1}\left(v_{-i}\right), \mu_{n}\right\}+1$ and define $v^{0} \equiv\left(v_{i}, v_{-i}\right)$. For each $r \in\{1,2, \ldots, n\}$, define $v^{r} \in V$ by:

$$
v_{k}^{r} \equiv\left\{\begin{array}{lr}
\pi, & k=A_{r}\left(v^{0}\right), \\
v_{k}^{r-1}, & \text { else. }
\end{array}\right.
$$

Observe that $v^{n}=v^{*}$. Observe also that at $v^{0}, w$ agents report more than $\pi$ and one agent reports $\pi$. We prove three claims, then conclude.

First, we claim that for each $r \in\{1,2, \ldots, n\}, \tau_{+}\left(v^{r}\right)=\tau_{-}\left(v^{r}\right)-\pi$. Indeed, let $r \in\{1,2, \ldots, n\}$. Since at $v^{r}$, less than $w$ agents report more than $\pi$ but more than $w$ agents bid at least $\pi$, and since $\pi \in\left(\mu_{w}, \mu_{w+1}\right)$, thus by the Bidding Lemma $\left|\varphi\left(v^{r}\right)\right|=w$, and moreover there are $j, j^{\prime} \in N$ such that $v_{j}^{r}=\pi=v_{j^{\prime}}^{r}, j \in \varphi\left(v^{r}\right)$, and $j^{\prime} \in N \backslash \varphi\left(v^{r}\right)$. By no-envy-in-trades for $j$ and $j^{\prime}, \pi+\tau_{+}\left(v^{r}\right)=\tau_{-}\left(v^{r}\right)$, as desired.

Second, we claim $\tau_{+}\left(v^{1}\right)=\tau_{+}\left(v^{0}\right)$. Since $v_{i}>B_{1}\left(v_{-i}\right)$, thus $i=A_{1}\left(v^{0}\right)$. Since $v_{i}>\mu_{n}$, thus by production efficiency $i \in \varphi\left(v^{0}\right)$, so $\tau_{+}\left(v^{0}\right)=\tau_{i}\left(v^{0}\right)$. By the Auction Lemma, either $i \in \varphi\left(v^{1}\right)$ and $\tau_{i}\left(v^{1}\right)=\tau_{+}\left(v^{0}\right)$ or $i \notin \varphi\left(v^{1}\right)$ and $\tau_{i}\left(v^{1}\right)=\tau_{+}\left(v^{0}\right)+\pi$. Thus either $\tau_{+}\left(v^{1}\right)=\tau_{+}\left(v^{0}\right)$ or $\tau_{-}\left(v^{1}\right)=\tau_{+}\left(v^{0}\right)+\pi$. By the first claim, in either case $\tau_{+}\left(v^{1}\right)=\tau_{+}\left(v^{0}\right)$, as desired.

Third, we claim that for each $r \in\{1,2, \ldots, n-1\}, \tau_{+}\left(v^{r+1}\right)=\tau_{+}\left(v^{r}\right)$. Indeed, let $r \in\{1,2, \ldots, n-1\}$ and define $j \equiv A_{r}\left(v^{r}\right)$. Since at $v_{-j}$ less than $w$ agents report more than $\pi$ but at least $w$ agents bid at least $\pi$, and since $\pi \in\left(\mu_{w}, \mu_{w+1}\right)$, thus $\pi\left(v_{-j}\right)=\pi$. We consider four exhaustive possibilities:

- $j \in \varphi\left(v^{r+1}\right)$ and $j \in \varphi\left(v^{r}\right)$. Then by strategy-proofness $\tau_{+}\left(v^{r+1}\right)=\tau_{+}\left(v^{r}\right)$ as desired.

- $j \in \varphi\left(v^{r+1}\right)$ and $j \notin \varphi\left(v^{r}\right)$. Then by the Auction Lemma $\tau_{+}\left(v^{r+1}\right)=\tau_{-}\left(v^{r}\right)-\pi$, so by the first claim $\tau_{+}\left(v^{r+1}\right)=\tau_{+}\left(v^{r}\right)$ as desired.

\footnotetext{
${ }^{14}$ This is an adaptation of a similar argument from Ohseto (2006).
} 
- $j \notin \varphi\left(v^{r+1}\right)$ and $j \in \varphi\left(v^{r}\right)$. Then by the Auction Lemma $\tau_{-}\left(v^{r+1}\right)=\tau_{+}\left(v^{r}\right)+\pi$, so by the first claim $\tau_{+}\left(v^{r+1}\right)=\tau_{+}\left(v^{r}\right)$ as desired.

- $j \notin \varphi\left(v^{r+1}\right)$ and $j \notin \varphi\left(v^{r}\right)$. Then by strategy-proofness $\tau_{-}\left(v^{r+1}\right)=\tau_{-}\left(v^{r}\right)$, so by the first claim $\tau_{+}\left(v^{r+1}\right)=\tau_{+}\left(v^{r}\right)$ as desired.

To conclude, by the first claim either $i \in \varphi\left(v^{*}\right)$ and $\tau_{i}\left(v^{*}\right)=\tau_{+}\left(v^{*}\right)$ or $i \notin \varphi\left(v^{*}\right)$ and $\tau_{i}\left(v^{*}\right)=\tau_{+}\left(v^{*}\right)+\pi$. Since at $v_{-i}^{*}$ less than $w$ agents report more than $\pi$ but at least $w$ agents bid at least $\pi$, and since $\pi \in\left(\mu_{w}, \mu_{w+1}\right)$, thus $\pi\left(v_{-i}^{*}\right)=\pi$. Since $v_{i}>\mu_{n}$, thus by production efficiency, $i \in \varphi\left(v_{i}, v_{-i}^{*}\right)$. Altogether, by the Auction Lemma $\tau_{i}\left(v_{i}, v_{-i}^{*}\right)=$ $\tau_{+}\left(v^{*}\right)$.

By the second and third claim, $\tau_{+}\left(v^{*}\right)=\tau_{+}\left(v^{n}\right)=\tau_{+}\left(v^{0}\right)=\tau_{+}(v)$. Since $v_{i}>\mu_{n}$, thus by production efficiency, $\tau_{i}(v)=\tau_{+}(v)=\tau_{+}\left(v^{*}\right)=\tau_{i}\left(v_{i}, v_{-i}^{*}\right)$. Since $i \in \varphi(v)$, $i \in \varphi\left(v_{i}, v_{-i}^{*}\right)$, and $\pi\left(v_{-i}\right)=\pi\left(v_{-i}^{*}\right)$, thus by the Auction Lemma, $\alpha_{i}\left(v_{-i}\right)=\alpha_{i}\left(v_{-i}^{*}\right)$.

Since $v_{-i} \in V_{-i}$ with $\pi\left(v_{-i}\right)=\pi$ was arbitrary, thus for each pair $v_{-i}, v_{-i}^{\prime} \in V_{-i}$ with $\pi\left(v_{-i}\right)=\pi=\pi\left(v_{-i}^{\prime}\right), \alpha_{i}\left(v_{-i}\right)=\alpha_{i}\left(v_{-i}^{*}\right)=\alpha_{i}\left(v_{-i}^{\prime}\right)$, as desired.

CASE 2: $\pi \in\left\{\mu_{1}, \mu_{2}, \ldots, \mu_{n}\right\}$. Since several marginal costs may be equal, define

$$
\begin{aligned}
& w_{-} \equiv \min \left\{w \in\{1,2, \ldots, n\} \mid \pi=\mu_{w}\right\}, \text { and } \\
& w_{+} \equiv \max \left\{w \in\{1,2, \ldots, n\} \mid \pi=\mu_{w}\right\} .
\end{aligned}
$$

We wish to partition $V_{-i}$ based on (i) the number $h$ of agents with reports higher than $\pi$, and (ii) the number $e$ of agents with reports equal to $\pi$. Formally, for each $v_{-i} \in V_{-i}$, define

$$
\begin{aligned}
H\left(v_{-i}\right) & \equiv\left|\left\{j \in N \backslash\{i\} \mid v_{j}>\pi\right\}\right|, \text { and } \\
E\left(v_{-i}\right) & \equiv\left|\left\{j \in N \backslash\{i\} \mid v_{j}=\pi\right\}\right| .
\end{aligned}
$$

For each pair $h, e \in\{0,1, \ldots, n-1\}$, define

$$
V_{-i}(h, e) \equiv\left\{v_{-i} \in V_{-i} \mid H\left(v_{-i}\right)=h \text { and } E\left(v_{-i}\right)=e\right\} .
$$

Note that these classes are mutually exclusive and collectively exhaustive, though some are empty (in particular, when $h+e>n-1$ ). Define the subcollection $\mathcal{V}$ of these classes over which price is constantly $\pi$ by:

$$
\mathcal{V} \equiv\left\{V_{-i}(h, e) \mid V_{-i}(h, e) \neq \emptyset \text { and } v_{-i} \in V_{-i}(h, e) \text { implies } \pi\left(v_{-i}\right)=\pi\right\}
$$

We claim that $\pi\left(v_{-i}\right)=\pi$ if and only if $v_{-i} \in \cup_{\mathcal{V}} V_{-i}(h, e)$.

Claim 1: For each $v_{-i} \in V_{-i}, \pi\left(v_{-i}\right)>\pi$ if and only if $H\left(v_{-i}\right) \geq w_{+}$.

$[\Rightarrow]$ We prove the contrapositive. Assume $w_{+}>H\left(v_{-i}\right)$. If $w_{+}=n$, then $\pi=\mu_{n} \geq$ $\pi\left(v_{-i}\right)$ as desired, so assume $n>w_{+}>H\left(v_{-i}\right)$.

If $w_{+}=n-1$, then $w_{+} \geq \Omega\left(v_{-i}\right)$. If $n-1>w_{+}$, then $\mu_{w_{+}+1}>\mu_{w_{+}} \geq B_{w_{+}}\left(v_{-i}\right) \geq$ $B_{w_{+}+1}\left(v_{-i}\right)$, so $w_{+} \geq \Omega\left(v_{-i}\right)$.

If $w_{+}>\Omega\left(v_{-i}\right)$, then $\pi=\mu_{w_{+}} \geq \mu_{\Omega\left(v_{-i}\right)+1} \geq \pi\left(v_{-i}\right)$.

If $w_{+}=\Omega\left(v_{-i}\right)$, then $\pi=\mu_{w_{+}} \geq B_{w_{+}}\left(v_{-i}\right)=B_{\Omega\left(v_{-i}\right)}\left(v_{-i}\right) \geq \pi\left(v_{-i}\right)$.

Altogether, $\pi\left(v_{-i}\right)>\pi$ implies $H\left(v_{-i}\right) \geq w_{+}$, as desired. 
$[\Leftarrow]$ Assume $H\left(v_{-i}\right) \geq w_{+}$. Then $B_{w_{+}}\left(v_{-i}\right)>\mu_{w_{+}}$, so $\Omega\left(v_{-i}\right) \geq w_{+}$.

If $\pi\left(v_{-i}\right)=B_{\Omega\left(v_{-i}\right)}\left(v_{-i}\right)$ and $\Omega\left(v_{-i}\right)=w_{+}$, then $\pi\left(v_{-i}\right)>\mu_{w_{+}}=\pi$ directly from above.

If $\pi\left(v_{-i}\right)=B_{\Omega\left(v_{-i}\right)}\left(v_{-i}\right)$ and $\Omega\left(v_{-i}\right)>w_{+}$, then $\pi\left(v_{-i}\right)=B_{\Omega\left(v_{-i}\right)}\left(v_{-i}\right) \geq \mu_{\Omega\left(v_{-i}\right)} \geq$ $\mu_{w_{+}+1}>\mu_{w_{+}}=\pi$.

If $\pi\left(v_{-i}\right)=\mu_{\Omega\left(v_{-i}\right)+1}$, then $\pi\left(v_{-i}\right)=\mu_{\Omega\left(v_{-i}\right)+1} \geq \mu_{w_{+}+1}>\mu_{w_{+}}=\pi$.

Altogether, $\pi\left(v_{-i}\right)>\mu_{w_{+}}=\pi$, as desired.

Claim 2: For each $v_{-i} \in V_{-i}, \pi>\pi\left(v_{-i}\right)$ if and only if $w_{-}-1>H\left(v_{-i}\right)+E\left(v_{-i}\right)$.

$[\Rightarrow]$ We prove the contrapositive. Assume $H\left(v_{-i}\right)+E\left(v_{-i}\right) \geq w_{-}-1$. Then $B_{w_{--1}}\left(v_{-i}\right) \geq$ $\mu_{w_{-}}>\mu_{w_{-}-1}$, so $\Omega\left(v_{-i}\right) \geq w_{-}-1$.

If $\pi\left(v_{-i}\right)=B_{\Omega\left(v_{-i}\right)}\left(v_{-i}\right)$ and $\Omega\left(v_{-i}\right)=w_{-}-1$, then $\pi\left(v_{-i}\right)>\mu_{w_{-}}=\pi$ directly from above.

If $\pi\left(v_{-i}\right)=B_{\Omega\left(v_{-i}\right)}\left(v_{-i}\right)$ and $\Omega\left(v_{-i}\right)>w_{-}-1$, then $\pi\left(v_{-i}\right)=B_{\Omega\left(v_{-i}\right)}\left(v_{-i}\right) \geq \mu_{\Omega\left(v_{-i}\right)} \geq$ $\mu_{w_{-}}=\pi$.

If $\pi\left(v_{-i}\right)=\mu_{\Omega\left(v_{-i}\right)+1}$, then $\pi\left(v_{-i}\right)=\mu_{\Omega\left(v_{-i}\right)+1} \geq \mu_{w_{-}}=\pi$.

Altogether, $\pi>\pi\left(v_{-i}\right)$ implies $w_{-}-1>H\left(v_{-i}\right)+E\left(v_{-i}\right)$, as desired.

$[\Leftarrow]$ Assume $w_{-}-1>H\left(v_{-i}\right)+E\left(v_{-i}\right)$. Then $\mu_{w_{-}}>B_{w_{-}-1}\left(v_{-i}\right) \geq B_{w_{-}}\left(v_{-i}\right)$, so $w_{-}-1 \geq \Omega\left(v_{-i}\right)$.

If $w_{-}-1=\Omega\left(v_{-i}\right)$, then $\pi=\mu_{w_{-}}>B_{w_{-}-1}\left(v_{-i}\right)=B_{\Omega\left(v_{-i}\right)}\left(v_{-i}\right) \geq \pi\left(v_{-i}\right)$.

If $w_{-}-1>\Omega\left(v_{-i}\right)$, then $\pi=\mu_{w_{-}}>\mu_{w_{-}-1} \geq \mu_{\Omega\left(v_{-i}\right)+1} \geq \pi\left(v_{-i}\right)$.

Altogether, $w_{-}-1>H\left(v_{-i}\right)+E\left(v_{-i}\right)$ implies $\pi>\pi\left(v_{-i}\right)$.

Claim 3: For each $h \in\{0,1, \ldots, n-1\}$ such that $V_{-i}(h, 0) \in \mathcal{V}, \alpha_{i}$ is constant on $V_{-i}(h, 0)$.

Let $h \in\{0,1, \ldots, n-1\}$ such that $V_{-i}(h, 0) \in \mathcal{V}$.

Subcase 1: $h=0$. Let $v_{-i}, v_{-i}^{\prime} \in V_{-i}(h, 0)$, and define $v, v^{\prime} \in V$ by $v \equiv$ $\left(\mu_{1}-1, v_{-i}\right)$ and $v^{\prime} \equiv\left(\mu_{1}-1, v_{-i}^{\prime}\right)$. Define $v^{0} \equiv v$, and for each $r \in\{1,2, \ldots, n\}$, recursively define $v^{r} \in V$ by $v^{r} \equiv\left(v_{r}^{\prime}, v_{-r}^{r-1}\right)$. For each $r \in\{0,1, \ldots, n\}$, at $v^{r}$ all agents report less than $\mu_{1}$ and lose, so all agents receive the same transfer. By strategy-proofness, for each $r \in\{1,2, \ldots, n\}, \tau_{r}\left(v^{r}\right)=\tau_{r}\left(v^{r-1}\right)$. Altogether, $\tau_{1}(v)=\tau_{1}\left(v^{0}\right)=\tau_{1}\left(v^{n}\right)=\tau_{1}\left(v^{\prime}\right)$, so by the Auction Lemma, $\alpha_{i}\left(v_{-i}\right)=\alpha_{i}\left(v_{-i}^{\prime}\right)$.

SUBCASE 2: $h>0$. First, we claim that for each $v_{-i} \in V_{-i}(h, 0)$, each $v_{i} \in V_{i}$ such that $\pi>v_{i}$, and each $j \in N$ such that $\pi>v_{j}, \pi_{j}\left(v_{-j}\right)=\pi$. Indeed, let $v_{-i} \in V_{-i}(h, 0)$, $v_{i} \in V_{i}$, and $j \in N$ satisfy these requirements. Since $E\left(v_{-i}\right)=0$, thus $B_{\Omega_{\left(v_{-i}\right)}}\left(v_{-i}\right) \neq \pi$, so $B_{\Omega\left(v_{-i}\right)}\left(v_{-i}\right)>\pi=\mu_{\Omega\left(v_{-i}\right)+1}$. By construction, $\mu_{\Omega\left(v_{-i}\right)+1}>B_{\Omega\left(v_{-i}\right)+1}\left(v_{-i}\right)$. Thus at $v$, there are $\Omega\left(v_{-i}\right)$ agents with bids over $\mu_{\Omega\left(v_{-i}\right)+1}$, and the others (including $i$ and $j$ ) have bids under $\mu_{\Omega\left(v_{-i}\right)+1}$. It follows that $\pi\left(v_{-j}\right)=\mu_{\Omega\left(v_{-i}\right)+1}=\pi$, as desired.

Next, we introduce a definition. For each $v_{-i} \in V_{-i}(h, 0)$, we say that $v_{-i}^{\prime \prime} \in V_{-i}(h, 0)$ is a swap of $v_{-i}$ if there are $j, k \in N \backslash\{i\}$ such that $v_{k}>\pi>v_{j}, v_{j}^{\prime \prime}>\pi>v_{k}^{\prime \prime}$, and

$$
v_{i^{\prime}}^{\prime \prime} \equiv\left\{\begin{array}{cc}
v_{j}^{\prime \prime}, & i^{\prime}=j, \\
v_{k}^{\prime \prime}, & i^{\prime}=k \\
v_{i^{\prime}}, & \text { else }
\end{array}\right.
$$


Observe that if $v_{-i}^{\prime \prime}$ is a swap of $v_{-i}$, then $v_{-i}^{\prime \prime} \in V_{-i}(h, 0)$ and $v_{-i}$ is a swap of $v_{-i}^{\prime \prime}$.

Second, we claim that for each $v_{-i} \in V_{-i}(h, 0)$ and each $v_{-i}^{\prime \prime}$ that is a swap of $v_{-i}$, $\alpha_{i}\left(v_{-i}\right)=\alpha_{i}\left(v_{-i}^{\prime \prime}\right)$. Indeed, let $v_{-i}, v_{-i}^{\prime \prime} \in V_{-i}(h, 0)$ and $j, k \in N \backslash\{i\}$ be as in the definition. Define $v_{-i}^{\prime} \in V_{-i}$ by $v_{-i}^{\prime} \equiv\left(v_{j}^{\prime \prime}, v_{-i, j}\right)$, and define $v, v^{\prime}, v^{\prime \prime} \in V$ by $v \equiv\left(\mu_{1}-1, v_{-i}\right)$, $v^{\prime} \equiv\left(\mu_{1}-1, v_{-i}^{\prime}\right)$, and $v^{\prime \prime} \equiv\left(\mu_{1}-1, v_{-i}^{\prime \prime}\right)$. As argued for the first claim, $\pi=\mu_{\Omega\left(v_{-i}\right)+1}$. By the first claim, $\pi\left(v_{-i}\right)=\pi\left(v_{-j}\right)=\pi$, so at $v$ both $i$ and $j$ lose, so by no-envy-in-trades $\tau_{j}(v)=\tau_{i}(v)$. By the Auction Lemma, $\tau_{j}\left(v^{\prime}\right)=\tau_{j}(v)-\pi=\tau_{i}(v)-\pi$. At $v^{\prime}$, there are $\Omega\left(v_{-i}\right)+1$ agents (including $j$ and $k$ ) with bids above $\pi$ and the others (including i) have bids below $\pi$. Since $\pi=\mu_{\Omega\left(v_{-i}\right)+1}$, thus $\pi\left(v_{-j}^{\prime}\right)=\pi\left(v_{-k}^{\prime}\right)=\pi$, so at $v^{\prime}$ both $j$ and $k$ win, so by no-envy-in-trades $\tau_{k}\left(v^{\prime}\right)=\tau_{j}\left(v^{\prime}\right)=\tau_{i}(v)-\pi$. By the Auction Lemma, $\tau_{k}\left(v^{\prime \prime}\right)=\tau_{k}\left(v^{\prime}\right)+\pi=\tau_{i}(v)$. By the first claim, $\pi\left(v_{-i}^{\prime \prime}\right)=\pi\left(v_{-k}^{\prime \prime}\right)=\pi$, so at $v^{\prime \prime}$ both $i$ and $k$ lose, so by no-envy-in-trades $\tau_{i}\left(v^{\prime \prime}\right)=\tau_{k}\left(v^{\prime \prime}\right)=\tau_{i}(v)$. Thus by the Auction Lemma, $\alpha_{i}\left(v_{-i}\right)=\alpha_{i}\left(v_{-i}^{\prime \prime}\right)$, as desired.

To conclude, let $v_{-i}, v_{-i}^{\prime} \in V_{-i}(h, 0)$. If $h \geq 1$, then with either one or two swaps from $v_{-i}$, we can arrive at $v_{-i}^{1}$ for which the first rank-index agent and his bid are as in $v_{-i}^{\prime}$. Similarly, if $h \geq 2$, with either one or two swaps from $v_{-i}^{1}$, we can arrive at $v_{-i}^{2}$ for which the first two rank-index agents and their bids are as in $v_{-i}^{\prime}$. Proceeding in this fashion, we can arrive at $v_{-i}^{h}$ for which the first $h$ rank-index agents and their bids are as in $v_{-i}^{\prime}$. By our second claim, $\alpha_{i}\left(v_{-i}\right)=\alpha_{i}\left(v_{-i}^{h}\right)$. At this point we use an argument similar to that in Subcase 1. Define $v^{h}, v^{\prime} \in V$ by $v^{h} \equiv\left(\mu_{1}-1, v_{-i}^{h}\right)$ and $v^{\prime} \equiv\left(\mu_{1}-1, v_{-i}^{\prime}\right)$. If there is $j \in N \backslash\{i\}$ with rank-index $h+1$ at $v_{-i}^{\prime}$, then $j$ bids less than $\pi$ at both $v^{h}$ and $v^{\prime}$, so by the first claim, both $i$ and $j$ face price $\pi$ and lose at both $v^{h}$ and $v^{\prime}$. By strategy-proofness $j$ receives the same transfer at both profiles, and by no-envy-in-trades $i$ receives the same transfer as $j$ at each profile, so altogether $i$ receives the same transfer at both profiles. By repeating this argument, $i$ receives the same transfer as we proceed from $v^{h}$ to $v^{\prime}$ by changing each agent's bid to match his bid at $v^{\prime}$ one at a time, so $\tau_{i}\left(v^{h}\right)=\tau_{i}\left(v^{\prime}\right)$. By the Auction Lemma, $\alpha_{i}\left(v_{-i}^{h}\right)=\alpha\left(v_{-i}^{\prime}\right)$, so altogether $\alpha_{i}\left(v_{-i}\right)=\alpha_{i}\left(v_{-i}^{\prime}\right)$, as desired.

Claim 4: For each pair $h, e \in\{0,1, \ldots, n-1\}$ such that $V_{-i}(h, e), V_{-i}(h, e+1) \in \mathcal{V}$, if $\alpha_{i}$ is constant on $V_{-i}(h, e)$, then $\alpha_{i}$ is constant on $V_{-i}(h, e+1) \cup V_{-i}(h, e)$.

Assume $V_{-i}(h, e), V_{-i}(h, e+1) \in \mathcal{V}$ and $\alpha_{i}$ is constant on $V_{-i}(h, e)$. Then there is $a \in \mathbb{R}$ such that for each $v_{-i} \in V_{-i}(h, e), \alpha_{i}\left(v_{-i}\right)=a$.

Let $v_{-i} \in V_{-i}(h, e+1)$. Since $v_{-i} \in V_{-i}(h, e+1) \in \mathcal{V}$, thus $\pi\left(v_{-i}\right)=\pi$. Define $v \in V$ by $\left(\pi, v_{-i}\right)$. By definition of $V_{-i}(h, e+1)$, there is $j \in N \backslash\{i\}$ such that $v_{j}=\pi$. Since $v_{i}=v_{j}$, thus $\pi\left(v_{-j}\right)=\pi\left(v_{-i}\right)=\pi$. By no-envy-in-trades, no matter whether both $i$ and $j$ win, or both lose, or one wins and the other loses, $\alpha_{i}\left(v_{-i}\right)=\alpha_{j}\left(v_{-j}\right)$.

For each $\epsilon>0$, define $v^{\epsilon} \in V$ by $\left(\pi-\epsilon, v_{-j}\right)$. Consider an arbitrary $v^{\epsilon}$. Since $v_{-i}^{\epsilon} \in V_{-i}(h, e)$, thus either $i$ wins and receives $a-\pi$ or $i$ loses and receives $a$. Moreover, $v_{-j}^{\epsilon}=v_{-j}$. By the Auction Lemma, $j$ loses and receives $\alpha_{j}\left(v_{-j}\right)$. If $i$ wins, then by no-envy-in-trades, $\alpha_{j}\left(v_{-j}\right) \geq(\pi-\epsilon)+(a-\pi)$ and $\pi+(a-\pi) \geq \alpha_{j}\left(v_{-j}\right)$. If $i$ loses, then by no-envy-in-trades, $\alpha_{j}\left(v_{-j}\right)=a$. Thus in both cases, $\alpha_{j}\left(v_{-j}\right) \in[a-\epsilon, a]$.

Since $\epsilon>0$ was arbitrary, thus $\alpha_{j}\left(v_{-j}\right)=a$, so $\alpha_{i}\left(v_{-i}\right)=a$. Since $v_{-i} \in V_{-i}(h, e+1)$ was arbitrary, thus $\alpha_{i}$ is constant on $V_{-i}(h, e+1) \cup V_{-i}(h, e)$, as desired.

Claim 5: For each pair $h, e \in\{0,1, \ldots, n-1\}$ such that $V_{-i}(h, e), V_{-i}(h-1, e+1) \in \mathcal{V}$, if $\alpha_{i}$ is constant on $V_{-i}(h, e)$, then $\alpha_{i}$ is constant on $V_{-i}(h-1, e+1) \cup V_{-i}(h, e)$. 
Assume $V_{-i}(h, e), V_{-i}(h-1, e+1) \in \mathcal{V}$ and $\alpha_{i}$ is constant on $V_{-i}(h, e)$. Then there is $a \in \mathbb{R}$ such that for each $v_{-i} \in V_{-i}(h, e), \alpha_{i}\left(v_{-i}\right)=a$.

Let $v_{-i} \in V_{-i}(h-1, e+1)$. Since $v_{-i} \in V_{-i}(h-1, e+1) \in \mathcal{V}$, thus $\pi\left(v_{-i}\right)=\pi$. Define $v \in V$ by $\left(\pi, v_{-i}\right)$. By definition of $V_{-i}(h-1, e+1)$, there is $j \in N \backslash\{i\}$ such that $v_{j}=\pi$. Since $v_{i}=v_{j}$, thus $\pi\left(v_{-j}\right)=\pi\left(v_{-i}\right)=\pi$. By no-envy-in-trades, no matter whether both $i$ and $j$ win, or both lose, or one wins and the other loses, $\alpha_{i}\left(v_{-i}\right)=\alpha_{j}\left(v_{-j}\right)$.

For each $\epsilon>0$, define $v^{\epsilon} \in V$ by $\left(\pi+\epsilon, v_{-j}\right)$. Consider an arbitrary $v^{\epsilon}$. Since $v_{-i}^{\epsilon} \in V_{-i}(h, e)$, thus either $i$ wins and receives $a-\pi$ or $i$ loses and receives $a$. Moreover, $v_{-j}^{\epsilon}=v_{-j}$. By the Auction Lemma, $j$ wins and receives $\alpha_{j}\left(v_{-j}\right)-\pi$. If $i$ wins, then by noenvy-in-trades, $\alpha_{j}\left(v_{-j}\right)=a$. If $i$ loses, then by no-envy-in-trades, $(\pi+\epsilon)+\left(\alpha_{j}\left(v_{-j}\right)-\pi\right) \geq$ $a$ and $a \geq \pi+\left(\alpha_{j}\left(v_{-j}\right)-\pi\right)$. Thus in both cases, $\alpha_{j}\left(v_{-j}\right) \in[a, a+\epsilon]$.

Since $\epsilon>0$ was arbitrary, thus $\alpha_{j}\left(v_{-j}\right)=a$, so $\alpha_{i}\left(v_{-i}\right)=a$. Since $v_{-i} \in V_{-i}(h-1, e+1)$ was arbitrary, thus $\alpha_{i}$ is constant on $V_{-i}(h-1, e+1) \cup V_{-i}(h, e)$, as desired.

Conclusion: The proof technique is illustrated by Figure 3. By Claim 1 and Claim 2, for each $v_{-i} \in V_{-i}, \pi\left(v_{-i}\right)=\pi$ if and only if

$$
v_{-i} \in \bigcup_{\mathcal{V}} V_{-i}(h, e)
$$

Moreover, $\mathcal{V}$ is the collection of classes $V_{-i}(h, e)$ such that

(i) $h \leq w_{+}-1$

(ii) $h+e \geq w_{-}-1$,

(iii) $h \geq 0$,

(iv) $e \geq 0$, and

(v) $h+e \leq n-1$.

Thus $V_{-i}\left(w_{-}-1,0\right) \in \mathcal{V}$ and $V_{-i}\left(w_{-}-2,0\right) \notin \mathcal{V}$. By Claim 3, $\alpha_{i}$ is constant on $V_{-i}\left(w_{-}-\right.$ $1,0)$, so by Claim $4, \alpha_{i}$ is constant on $\cup\left\{V_{-i}\left(w_{-}-1, e\right) \in \mathcal{V}\right\}$, so by Claim $5, \alpha_{i}$ is constant on $\cup\left\{V_{-i}(h, e) \in \mathcal{V} \mid w_{-}-1 \geq h\right\}$.

Suppose there are $h, e \in\{0,1, \ldots, n-1\}$ such that $V_{-i}(h, e) \in \mathcal{V}$ and $h>w_{-}-1$. Then $V_{-i}(h, 0) \in \mathcal{V}$. By Claim 3, $\alpha_{i}$ is constant on $V_{-i}(h, 0)$, so by Claim $4 \alpha_{i}$ is constant on $V_{-i}(h, 0) \cup V_{-i}(h, e)$. Moreover, for each $m \in\left\{0,1, \ldots, h-\left(w_{-}-1\right)\right\}, V(h-m, e+m) \in \mathcal{V}$, so by Claim $5 \alpha_{i}$ is constant on $V_{-i}(h, 0) \cup V_{-i}\left(w_{-}-1, h-\left(w_{-}-1\right)\right)$. Thus by the previous paragraph, $\alpha_{i}$ is constant on $V_{-i}\left(w_{-}-1,0\right) \cup V_{-i}(h, e)$.

Altogether, $\alpha_{i}$ is constant on $\left\{V_{-i}(h, e) \in \mathcal{V}\right\}$. Thus for each pair $v_{-i}, v_{-i}^{\prime} \in V_{-i}$ such that $\pi\left(v_{-i}\right)=\pi\left(v_{-i}^{\prime}\right), \alpha_{i}\left(v_{-i}\right)=\alpha_{i}\left(v_{-i}^{\prime}\right)$, as desired.

\section{Appendix 7}

In this appendix, we prove Theorem 2 .

Theorem 2: In the object setting, a rule $(\tau, \varphi)$ satisfies production efficiency, strategyproofness, and no-envy-in-trades if and only it is a citizen-shareholder rule.

ProOF: We establish both implications.

$[\Rightarrow]$ Let $(\tau, \varphi)$ satisfy the axioms. By the Consolation Lemma, then $(\tau, \varphi)$ is a peerconsolation rule with peer-consolation schedules $\left(\alpha_{i}\right)$ such that for each $i \in N$ and each 


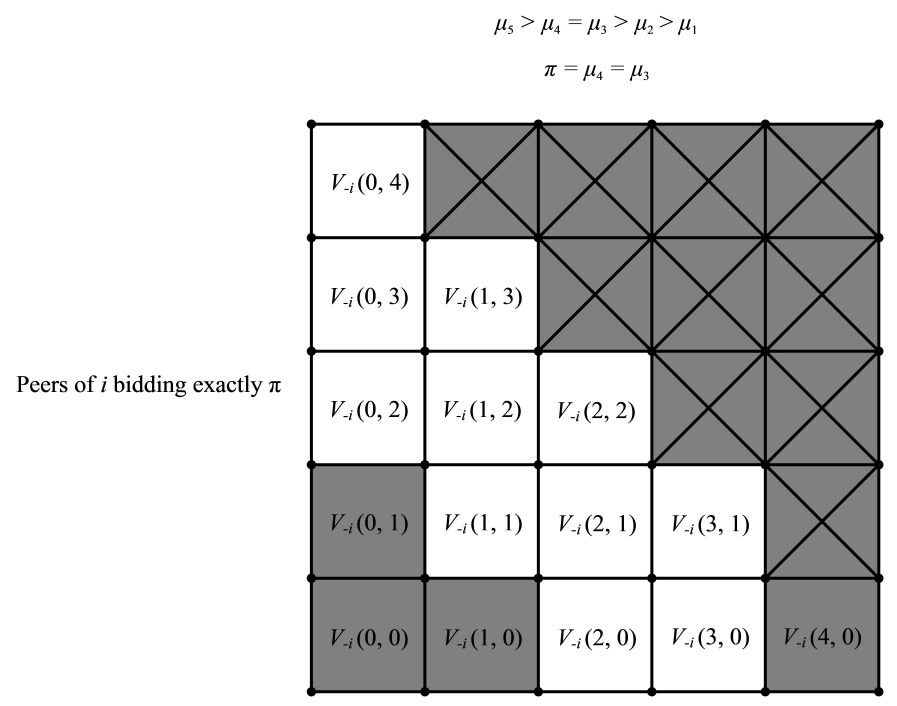

Peers of $i$ bidding higher than $\pi$

Figure 3: In this example, there are five agents, $\mu_{5}>\mu_{4}=\mu_{3}>\mu_{2}>\mu_{1}$, and $\pi=\mu_{4}=\mu_{3}$. We have selected an agent $i$, and wish to show that $\alpha_{i}$ is constant across the peer-profiles in $V_{-i}$ that offer him price $\pi$. The horizontal axis measures the number of peers who bid higher than $\pi$ and the vertical axis measures the number of peers who bid exactly $\pi$. Each box in the grid $V_{-i}(h, e)$ is the corresponding class of peer profiles; those crossed out are empty. By Claim 1 and Claim 2, $\mathcal{V}$ is the collection of white (unshaded) boxes, and the profiles in these boxes are precisely those that offer price $\pi$. By Claim $3, \alpha_{i}$ is constant over each white box in the bottom row. By Claim 4, if $\alpha_{i}$ is constant over one white box with a second white box above it, then $\alpha_{i}$ is constant over both. By Claim 5, if $\alpha_{i}$ is constant over one white box with a second white box directly to its upper-left, then $\alpha_{i}$ is constant over both. These observations together imply that $\alpha_{i}$ is constant over the white boxes. 
pair $v_{-i}, v_{-i}^{\prime} \in V_{-i}$

$$
\pi\left(v_{-i}\right)=\pi\left(v_{-i}^{\prime}\right) \text { implies } \alpha_{i}\left(v_{-i}\right)=\alpha_{i}\left(v_{-i}^{\prime}\right) .
$$

Thus there are functions $\beta_{i}:\left[\mu_{1}, \mu_{n}\right] \rightarrow \mathbb{R}$ such that for each $i \in N$ and each $v_{-i} \in V_{-i}$,

(i) $\left(\tau_{i}(v), \varphi_{i}(v)\right)$ is either $\left(\beta_{i}\left(\pi\left(v_{-i}\right)\right)-\pi\left(v_{-i}\right), 1\right)$ or $\left(\beta_{i}\left(\pi\left(v_{-i}\right)\right), 0\right)$,

(ii) $v_{i}>\pi\left(v_{-i}\right)$ implies $i \in \varphi(v)$, and

(iii) $\pi\left(v_{-i}\right)>v_{i}$ implies $i \notin \varphi(v)$.

Let $\pi \in\left[\mu_{1}, \mu_{n}\right]$, and let $v \in V$ be the profile where each agent reports $\pi$. Assume, by way of contradiction, there is $i \in N$ such that $\pi\left(v_{-i}\right) \neq \pi$. Then $B_{\Omega\left(v_{-i}\right)}\left(v_{-i}\right)>\mu_{\Omega\left(v_{-i}\right)+1}=$ $\pi\left(v_{-i}\right)$. But if $n-1 \geq \Omega\left(v_{-i}\right)+1$, then $B_{\Omega\left(v_{-i}\right)+1}\left(v_{-i}\right) \geq \mu_{\Omega\left(v_{-i}\right)+1}$, which is impossible by definition of $\Omega\left(v_{-i}\right)$. Thus $\Omega\left(v_{-i}\right)=n-1$. But then $B_{n-1}\left(v_{-i}\right)>\mu_{n}$, contradicting that each agent reports $\pi \in\left[\mu_{1}, \mu_{n}\right]$. Altogether, at $v$, each winner $i$ receives $\beta_{i}(\pi)-\pi$ and each loser $j$ receives $\beta_{j}(\pi)$. By no-envy-in-trades, for each pair $i, j \in N, \beta_{i}(\pi)=\beta_{j}(\pi)$. Since $\pi$ was arbitrary, for each pair $i, j \in N, \beta_{i}=\beta_{j}$.

Thus there is $\alpha_{0}:\left[\mu_{1}, \mu_{n}\right] \rightarrow \mathbb{R}$ such that for each $i \in N$ and each $v_{-i} \in V_{-i}$, $\left(\tau_{i}(v), \varphi_{i}(v)\right)$ is either $\left(\alpha_{0}\left(\pi\left(v_{-i}\right)\right)-\pi\left(v_{-i}\right), 1\right)$ or $\left(\alpha_{0}\left(\pi\left(v_{-i}\right)\right), 0\right)$.

Let $w \in\{1,2, \ldots, n-1\}$ such that $\mu_{w+1}>\mu_{w}$ and let $\pi, \pi^{\prime} \in\left[\mu_{w}, \mu_{w+1}\right]$ such that $\pi^{\prime}>\pi$. Let $v$ be a profile where $w$ agents report $\pi^{\prime}$ and the others report $\pi$; note that there are agents in both groups. Let $i$ be an agent with $v_{i}=\pi^{\prime}$ and let $j$ be an agent with $v_{j}=\pi$. It can be easily verified that $\pi\left(v_{-i}\right)=\pi$ and $\pi\left(v_{-j}\right)=\pi^{\prime}$; thus $i$ wins and receives $\alpha_{0}(\pi)-\pi$ and $j$ loses and receives $\alpha_{0}\left(\pi^{\prime}\right)$. By no-envy-in-trades, $\pi^{\prime}+\left(\alpha_{0}(\pi)-\pi\right) \geq \alpha_{0}\left(\pi^{\prime}\right)$ and $\alpha_{0}\left(\pi^{\prime}\right) \geq \pi+\left(\alpha_{0}(\pi)-\pi\right)$. Thus

$$
\begin{aligned}
1 & \geq \frac{\alpha_{0}\left(\pi^{\prime}\right)-\alpha_{0}(\pi)}{\pi^{\prime}-\pi}, \text { and } \\
\alpha_{0}\left(\pi^{\prime}\right) & \geq \alpha_{0}(\pi) .
\end{aligned}
$$

Since $\pi, \pi^{\prime}$ were arbitrary, thus $\alpha_{0}$ is Lipschitz-continuous with the desired restriction on $\left[\mu_{w}, \mu_{w+1}\right]$. Since $w$ was arbitrary, thus $\alpha_{0}$ is Lipschitz-continuous with the desired restriction on $\left[\mu_{1}, \mu_{n}\right]$, so it is a social-dividend schedule. Altogether, $(\tau, \varphi)$ is a citizenshareholder rule.

$[\Leftarrow]$ Suppose $(\tau, \varphi)$ is a citizen-shareholder rule, with $\alpha_{0}$ its social-dividend schedule. By the Bidding Lemma and the Auction Lemma, $(\tau, \varphi)$ satisfies production efficiency and strategy-proofness. Assume, by way of contradiction, $(\tau, \varphi)$ violates no-envy-in-trades. Then there are $v \in V$ and $i, j \in N$ such that

$$
v_{i} \varphi_{j}(v)+\tau_{j}(v)>v_{i} \varphi_{i}(v)+\tau_{i}(v) .
$$

This is clearly impossible if both agents win or if both agents lose.

If $i$ wins and $j$ loses, then by the Winner-Loser Prices Lemma, $v_{i} \geq \pi\left(v_{-j}\right) \geq \pi\left(v_{-i}\right) \geq$ $v_{j}$, so as $\alpha_{0}$ is a social-dividend schedule,

$$
\begin{aligned}
v_{i} \varphi_{i}(v)+\tau_{i}(v) & =v_{i}+\left(\alpha_{0}\left(\pi\left(v_{-i}\right)\right)-\pi\left(v_{-i}\right)\right) \\
& =\alpha_{0}\left(\pi\left(v_{-i}\right)\right)+\left(v_{i}-\pi\left(v_{-i}\right)\right) \\
& \geq \alpha_{0}\left(\pi\left(v_{-i}\right)\right)+\left(\alpha_{0}\left(v_{i}\right)-\alpha_{0}\left(\pi\left(v_{-i}\right)\right)\right) \\
& \geq \alpha_{0}\left(\pi\left(v_{-i}\right)\right)+\alpha_{0}\left(\pi\left(v_{-j}\right)\right)-\alpha_{0}\left(\pi\left(v_{-i}\right)\right) \\
& =\alpha_{0}\left(\pi\left(v_{-j}\right)\right) \\
& =v_{i} \varphi_{j}(v)+\tau_{j}(v),
\end{aligned}
$$


as desired.

If $i$ loses and $j$ wins, then by the Winner-Loser Prices Lemma, $v_{j} \geq \pi\left(v_{-i}\right) \geq \pi\left(v_{-j}\right) \geq$ $v_{i}$, so as $\alpha_{0}$ is a social-dividend schedule,

$$
\begin{aligned}
v_{i} \varphi_{i}(v)+\tau_{i}(v) & =\alpha_{0}\left(\pi\left(v_{-i}\right)\right) \\
& \geq \alpha_{0}\left(\pi\left(v_{-j}\right)\right. \\
& =v_{i}+\left(\alpha_{0}\left(\pi\left(v_{-j}\right)-v_{i}\right)\right. \\
& \geq v_{i}+\left(\alpha_{0}\left(\pi\left(v_{-j}\right)\right)-\pi\left(v_{-j}\right)\right) \\
& =v_{i} \varphi_{j}(v)+\tau_{j}(v),
\end{aligned}
$$

as desired.

\section{Appendix 8}

In this appendix, we prove Theorem 3 .

THEOREM 3: In the object setting, a rule $(\tau, \varphi)$ satisfies no-deficit, production efficiency, strategy-proofness and no-envy-in-trades if and only it is a cost-constrained citizenshareholder rule.

Proof: We establish both implications.

$[\Rightarrow]$ Let $(\tau, \varphi)$ satisfy the axioms. By Theorem 2, $(\tau, \varphi)$ is a citizen-shareholder rule with social-dividend schedule $\alpha_{0}$. We verify that $\alpha_{0}$ satisfies the restrictions:

(i) For each $i \in N$, define $v_{i} \equiv-1$. By the Bidding Lemma, $\varphi(v)=\emptyset$. Moreover, for each $i \in N, \Omega\left(v_{-i}\right)=0$, so $\pi\left(v_{-i}\right)=\mu_{1}$. Thus by no-deficit,

$$
\begin{aligned}
0 & =-C(\varphi(v)) \\
& \geq \sum \tau_{i}(v) \\
& =n \alpha_{0}\left(\mu_{1}\right),
\end{aligned}
$$

so $\alpha_{0}\left(\mu_{1}\right) \leq 0$, as desired.

(ii) Let $w \in\{1,2, \ldots, n-1\}$. Define $v \in V$ by:

$$
v_{i} \equiv\left\{\begin{array}{lr}
\mu_{n}+1, & i \in\{1,2, \ldots, w\} \\
-1, & \text { else. }
\end{array}\right.
$$

By the Bidding Lemma, $\varphi(v)=\{1,2, \ldots, w\}$. Moreover, $\Omega(v)=w, \pi_{+}(v)=\mu_{w}$, and $\pi_{-}(v)=\mu_{w+1}$, so by the Winner-Loser Prices Lemma, so for each $i \in \varphi(v), \pi\left(v_{-i}\right)=\mu_{w}$ and for each $i \in N \backslash \varphi(v), \pi\left(v_{-i}\right)=\mu_{w+1}$. Thus by no-deficit,

$$
\begin{aligned}
-C(w) & =-C(\varphi(v)) \\
& \geq \sum \tau_{i}(v) \\
& =w\left(\alpha_{0}\left(\mu_{w}\right)-\mu_{w}\right)+(n-w) \alpha_{0}\left(\mu_{w+1}\right),
\end{aligned}
$$


so $(n-w) \alpha_{0}\left(\mu_{w+1}\right) \leq\left(w \mu_{w}-C(w)\right)-w \alpha_{0}\left(\mu_{w}\right)$, as desired.

(iii) For each $i \in N$, define $v_{i} \equiv \mu_{n}+1$. By the Bidding Lemma, $\varphi(v)=N$. Moreover, for each $i \in N, \Omega\left(v_{-i}\right)=n-1$, so $\pi\left(v_{-i}\right)=\mu_{n}$. Thus by no-deficit,

$$
\begin{aligned}
-C(n) & =-C(\varphi(v)) \\
& \geq \sum \tau_{i}(v) \\
& =n\left(\alpha_{0}\left(\mu_{n}\right)-\mu_{n}\right),
\end{aligned}
$$

so $\alpha_{0}\left(\mu_{n}\right) \leq \frac{n \mu_{n}-C(n)}{n}$, as desired

$[\Leftarrow]$ Let $(\tau, \varphi)$ be a cost-constrained citizen-shareholder rule with social-dividend schedule $\alpha_{0}$. By Theorem 2, $(\tau, \varphi)$ satisfies production efficiency, strategy-proofness, and no-envyin-trades.

Let $v \in V$. Define $w \equiv|\varphi(v)|$, define $\pi_{+} \equiv \pi_{+}(v)$, and define $\pi_{-} \equiv \pi_{-}(v)$.

CASE 1: $w=0$. By the Bidding Lemma, $\mu_{1} \geq B_{1}(v)$. If $\Omega(v)=0$, then $\pi_{-}=\mu_{1}$. If $\Omega(v)>0$, then $\mu_{1} \geq B_{1}(v) \geq B_{\Omega(v)}(v) \geq \pi_{-}$, so $\pi_{-}=\mu_{1}$. Thus in both cases, $\pi_{-}=\mu_{1}$, so by (i),

$$
\begin{aligned}
\sum \tau_{i}(v) & =n \alpha_{0}\left(\mu_{1}\right) \\
& \leq 0 \\
& =-C(\varphi(v))
\end{aligned}
$$

as desired.

CASE $2: w \in\{1,2, \ldots, n-1\}$ and $w<\Omega(v)$. Then $w+1 \leq \Omega(v)$, so by the Bidding Lemma and the Winner-Loser Prices Lemma, $\mu_{w+1} \geq B_{w+1}(v) \geq B_{\Omega(v)}(v) \geq \pi_{-} \geq \pi_{+} \geq \mu_{\Omega(v)} \geq$ $\mu_{w+1}$, so $\mu_{w+1}=\pi_{-}=\pi_{+}$.

If $\mu_{w+1}=\mu_{w}$, then $\left(\alpha_{0}\left(\mu_{w+1}\right)-\mu_{w+1}\right)=\left(\alpha_{0}\left(\mu_{w}\right)-\mu_{w}\right)$. If $\mu_{w+1}>\mu_{w}$, then since $\alpha_{0}$ is a social-dividend schedule, $\frac{\alpha_{0}\left(\mu_{w+1}\right)-\alpha_{0}\left(\mu_{w}\right)}{\mu_{w+1}-\mu_{w}} \leq 1$, so $\alpha_{0}\left(\mu_{w+1}\right)-\alpha_{0}\left(\mu_{w}\right) \leq \mu_{w+1}-\mu_{w}$, so $\left(\alpha_{0}\left(\mu_{w+1}\right)-\mu_{w+1}\right) \leq\left(\alpha_{0}\left(\mu_{w}\right)-\mu_{w}\right)$. Thus in both cases, $\left(\alpha_{0}\left(\mu_{w+1}\right)-\mu_{w+1}\right) \leq\left(\alpha_{0}\left(\mu_{w}\right)-\right.$ $\left.\mu_{w}\right)$, so by (ii),

$$
\begin{aligned}
\sum \tau_{i}(v) & =w\left(\alpha_{0}\left(\pi_{+}\right)-\pi_{+}\right)+(n-w) \alpha_{0}\left(\pi_{-}\right) \\
& =w\left(\alpha_{0}\left(\mu_{w+1}\right)-\mu_{w+1}\right)+(n-w) \alpha_{0}\left(\mu_{w+1}\right) \\
& \leq w\left(\alpha_{0}\left(\mu_{w}\right)-\mu_{w}\right)+(n-w) \alpha_{0}\left(\mu_{w+1}\right) \\
& \leq-C(w) \\
& =-C(\varphi(v)),
\end{aligned}
$$

as desired.

CASE 3: $w \in\{1,2, \ldots, n-1\}$ and $w=\Omega(v)$. Then $\mu_{w+1}=\mu_{\Omega(v)+1} \geq \pi_{-}$and $\pi_{+} \geq$ $\mu_{\Omega(v)}=\mu_{w}$. 
If $\pi_{+}=\mu_{w}$, then $\left(\alpha_{0}\left(\pi_{+}\right)-\pi_{+}\right)=\left(\alpha_{0}\left(\mu_{w}\right)-\mu_{w}\right)$. If $\pi_{+}>\mu_{w}$, then since $\alpha_{0}$ is a social-dividend schedule, $\frac{\alpha_{0}\left(\pi_{+}\right)-\alpha_{0}\left(\mu_{w}\right)}{\pi_{+}-\mu_{w}} \leq 1$, so $\alpha_{0}\left(\pi_{+}\right)-\alpha_{0}\left(\mu_{w}\right) \leq \pi_{+}-\mu_{w}$, so $\left(\alpha_{0}\left(\pi_{+}\right)-\pi_{+}\right) \leq\left(\alpha_{0}\left(\mu_{w}\right)-\mu_{w}\right)$. Thus in both cases, $\left(\alpha_{0}\left(\pi_{+}\right)-\pi_{+}\right) \leq\left(\alpha_{0}\left(\mu_{w}\right)-\mu_{w}\right)$.

Since $\alpha_{0}$ is a social-dividend schedule, it is non-decreasing, so $\alpha_{0}\left(\pi_{-}\right) \leq \alpha_{0}\left(\mu_{w+1}\right)$. Thus by (ii),

$$
\begin{aligned}
\sum \tau_{i}(v) & =w\left(\alpha_{0}\left(\pi_{+}\right)-\pi_{+}\right)+(n-w) \alpha_{0}\left(\pi_{-}\right) \\
& \leq w\left(\alpha_{0}\left(\mu_{w}\right)-\mu_{w}\right)+(n-w) \alpha_{0}\left(\mu_{w+1}\right) \\
& \leq-C(w) \\
& =-C(\varphi(v))
\end{aligned}
$$

as desired.

CASE 4: $w=n$. By the Bidding Lemma, $B_{n}(v) \geq \mu_{n}$, so $\Omega(v)=n$, so $\pi_{+}=\mu_{n}$. Thus by (iii),

$$
\begin{aligned}
\sum \tau_{i}(v) & =n\left(\alpha_{0}\left(\mu_{n}\right)-\mu_{n}\right) \\
& \leq-C(n) \\
& =-C(\varphi(v))
\end{aligned}
$$

as desired.

Thus in each case, $\sum \tau_{i}(v) \leq-C(\varphi(v))$. Since $v \in V$ was arbitrary, thus $(\tau, \varphi)$ satisfies no-deficit, as desired.

\section{Appendix 9}

In this appendix, we prove Theorem 4.

To prove this theorem, it is convenient to be able to abstract from the problem of selecting winners altogether. Let us say that a rule is standard if it first selects as many winners as possible subject to production efficiency, then chooses the winners according to their rank-indices. Formally, define:

$$
\varphi^{\oplus}(v) \equiv\left\{A_{r}(v) \in N \mid \Omega(v) \geq r\right\} .
$$

Definition: A rule $(\tau, \varphi)$ is standard if $\varphi=\varphi^{\oplus}$, in which case we also say $\varphi$ is standard.

Note that each standard rule indeed satisfies production efficiency. Next, we define an operator that associates each rule with the standard rule that is Pareto-indifferent to it, called its standardization: ${ }^{15}$

${ }^{15}$ The reader may wonder why we did not abstract from this problem earlier; we did not do so because even though our axioms are preserved by standardization, establishing this fact relies crucially on Theorem 2. To put it another way, standardness can be viewed as a strong continuity axiom for the winner policy, and it is not at all obvious that this requirement can be imposed without loss of generality. 
Definition: For each rule $(\tau, \varphi)$, the standardization of $(\tau, \varphi)$ is the rule $\left(\tau^{\oplus}, \varphi^{\oplus}\right)$, where

$$
\tau_{i}^{\oplus}(v) \equiv\left\{\begin{array}{lr}
\tau_{i}(v)-v_{i}, & i \in \varphi^{\oplus}(v) \backslash \varphi(v), \\
\tau_{i}(v)+v_{i}, & i \in \varphi(v) \backslash \varphi\left(v^{\oplus}\right) \\
\tau_{i}(v), & \text { else }
\end{array}\right.
$$

We now prove the theorem:

THEOREM 4: In the object setting,

(I) a rule $(\tau, \varphi)$ (i) satisfies no-deficit, production efficiency, strategy-proofness, and no-envy-in-trades, and (ii) is not Pareto-dominated by another such rule, if and only if it is a cost-constrained citizen-shareholder rule with an unrelenting social-dividend schedule whose marginal cost award profile belongs to $\mathcal{F}$.

(II) a rule $(\tau, \varphi)$ (i) satisfies no-deficit, production efficiency, voluntarism, strategyproofness, and no-envy-in-trades, and (ii) is not Pareto-dominated by another such rule, if and only if it is a cost-constrained citizen-shareholder rule with an unrelenting socialdividend schedule whose marginal cost award profile belongs to $\mathcal{F}^{V}$.

Proof: By Theorem 3 and the definition of a cost-constrained social-dividend schedule, a rule $(\tau, \varphi)$ satisfies the (I) axioms if and only if it is a citizen-shareholder rule with a social-dividend schedule whose marginal cost award profile belongs to $\mathcal{A}$. We claim such a rule is voluntary if and only if its marginal cost award profile belongs to $\mathcal{A}^{V}$.

Indeed, let $(\tau, \varphi)$ satisfy the (I) axioms, let $\alpha_{0}$ be its social-dividend schedule, and let $a \in \mathcal{A}$ be its marginal cost award profile. Since $a \in \mathcal{A}$, thus $a_{1} \leq 0$. If $a \in \mathcal{A}^{V}$, then $a_{0}=0$, so since $\alpha_{0}$ is non-decreasing, $\alpha_{0}$ only makes non-negative assignments. Thus at each profile, each agent receives something at least as good as losing with a nonnegative social-dividend award, so $(\tau, \varphi)$ is voluntary. If $a \notin \mathcal{A}^{V}$, then $a_{0}<0$, so when each agent reports valuation -1 , each agent loses and receives $a_{0}<0$, so $(\tau, \varphi)$ is not voluntary.

From here, we prove three claims, then conclude.

Claim 1: Each rule is Pareto-indifferent to its standardization.

Let $i \in N$ and let $v \in V$. If $\left(\tau_{i}^{\oplus}, \varphi^{\oplus}(v)\right)=\left(\tau_{i}(v), \varphi(v)\right)$, then we are done. Otherwise, there is $t_{i} \in \mathbb{R}$ such that $\left\{\left(\tau_{i}^{\oplus}, \varphi^{\oplus}(v)\right),\left(\tau_{i}(v), \varphi(v)\right)\right\}=\left\{\left(t_{i}, 0\right),\left(t_{i}-v_{i}, 1\right)\right\}$. With preferences $v_{i}, i$ is indifferent between the two. Since $i \in N$ was arbitrary, all agents are indifferent between the two rules at $v$. Since $v \in V$ was arbitrary, thus $\left(\tau^{\oplus}, \varphi^{\oplus}\right)$ is Pareto-indifferent to $(\tau, \varphi)$.

Claim 2: Let $\left(\tau^{*}, \varphi^{*}\right)$ and $(\tau, \varphi)$ be standard citizen-shareholder rules with socialdividend schedules $\alpha_{0}^{*}$ and $\alpha_{0}$, respectively. If (i) $\alpha_{0}^{*}$ and $\alpha_{0}$ share marginal cost award profile $a$, and (ii) $\alpha_{0}^{*}$ is unrelenting while $\alpha_{0}$ is not, then $\alpha_{0}^{*}$ strictly Pareto-dominates $\alpha_{0}$.

Let $\left(\tau^{*}, \varphi^{*}\right),(\tau, \varphi), \alpha_{0}^{*}, \alpha_{0}$, and $a$ satisfy the hypotheses. Then $a \in \mathcal{A}$. Let $\pi \in\left[\mu_{1}, \mu_{n}\right]$.

We claim that $\alpha_{0}(\pi) \leq \alpha_{0}^{*}(\pi)$. Indeed, if $\pi \in\left\{\mu_{1}, \mu_{2}, \ldots, \mu_{n}\right\}$, then $\alpha_{0}(\pi)=\alpha_{0}^{*}(\pi)$. Otherwise, there is $w \in\{1,2, \ldots, n\}$ and $\delta \in\left(0, \mu_{w+1}-\mu_{w}\right)$ such that $\pi=\mu_{w}+\delta$. If $\delta<a_{w+1}-a_{w}$, then $\alpha_{0}(\pi) \leq \alpha_{0}^{*}(\pi)$; otherwise $\alpha_{0}(\pi)-\alpha_{0}\left(\mu_{w}\right)>\alpha_{0}^{*}(\pi)-a_{w}=\pi-\mu_{w}$, contradicting $a \in \mathcal{A}$. If $\delta \geq a_{w+1}-a_{w}$, then $\alpha_{0}(\pi) \leq \alpha_{0}^{*}(\pi)$; otherwise $\alpha_{0}\left(\mu_{w+1}\right)-\alpha_{0}(\pi)<$ $a_{w+1}-\alpha_{0}^{*}(\pi)=0$, contradicting $a \in \mathcal{A}$. Thus in all cases, $\alpha_{0}(\pi) \leq \alpha_{0}^{*}(\pi)$, as desired. 
Since $\pi \in\left[\mu_{1}, \mu_{n}\right]$ was arbitrary, thus for each $\pi \in\left[\mu_{1}, \mu_{n}\right], \alpha_{0}(\pi) \leq \alpha_{0}^{*}(\pi)$. Moreover, since $\alpha$ is not unrelenting, $\alpha_{0} \neq \alpha_{0}^{*}$, so there is some $\pi \in\left[\mu_{1}, \mu_{n}\right]$ such that $\alpha_{0}(\pi)<\alpha_{0}^{*}(\pi)$. From here, since $\left(\tau^{*}, \varphi^{*}\right)$ and $(\tau, \varphi)$ are standard, it is straightforward to show that $\left(\tau^{*}, \varphi^{*}\right)$ strictly Pareto-dominates $(\tau, \varphi)$.

Claim 3: Let $\left(\tau^{*}, \varphi^{*}\right)$ and $(\tau, \varphi)$ be standard citizen-shareholder rules with unrelenting social-dividend schedules $\alpha_{0}^{*}$ and $\alpha_{0}$, respectively, and let $a^{*}$ and $a$ be their respective marginal cost award profiles. Then $\left(\tau^{*}, \varphi^{*}\right)$ strictly Pareto-dominates $(\tau, \varphi)$ if and only if $a^{*}>a$.

Let $\left(\tau^{*}, \varphi^{*}\right),(\tau, \varphi), \alpha_{0}^{*}, \alpha_{0}, a^{*}$, and $a$ satisfy the hypotheses. Then $a^{*}, a \in \mathcal{A}$.

If $a^{*}>a$, then let $\pi \in\left[\mu_{1}, \mu_{n}\right]$. Then there are $w \in\{1,2, \ldots, n\}$ and $\delta \in\left[0, \mu_{w+1}-\mu_{w}\right]$ such that $\pi=\mu_{w}+\delta$. Since $\alpha_{0}^{*}\left(\mu_{w}\right)+\delta=a_{w}^{*}+\delta \geq a_{w}+\delta=\alpha_{0}\left(\mu_{w}\right)+\delta$ and $\alpha_{0}^{*}\left(\mu_{w+1}\right)=$ $a_{w+1}^{*} \geq a_{w+1}=\alpha_{0}\left(\mu_{w+1}\right)$, and since $\alpha_{0}^{*}$ and $\alpha_{0}$ are unrelenting, thus $\alpha_{0}^{*}(\pi) \geq \alpha_{0}(\pi)$. Since $\pi \in\left[\mu_{1}, \mu_{n}\right]$ was arbitrary, thus for each $\pi \in\left[\mu_{1}, \mu_{n}\right], \alpha_{0}(\pi) \leq \alpha_{0}^{*}(\pi)$. Moreover, since $a^{*}>a$, there is $w \in\{1,2, \ldots, n\}$ such that $\alpha_{0}\left(\mu_{w}\right)<\alpha_{0}^{*}\left(\mu_{w}\right)$. From here, since $\left(\tau^{*}, \varphi^{*}\right)$ and $(\tau, \varphi)$ are standard, it is straightforward to show that $\left(\tau^{*}, \varphi^{*}\right)$ strictly Pareto-dominates $(\tau, \varphi)$.

If it is not the case that $a^{*}>a$, then either (i) $\alpha_{0}=\alpha_{0}^{*}$ or (ii) there is $w \in\{1,2, \ldots, n\}$ such that $\alpha_{0}\left(\mu_{w}\right)>\alpha_{0}^{*}\left(\mu_{w}\right)$. If $(\mathrm{i})$, then since $\left(\tau^{*}, \varphi^{*}\right)$ and $(\tau, \varphi)$ are standard, they are equal, so neither strictly Pareto-dominates the other. If (ii), then since $\left(\tau^{*}, \varphi^{*}\right)$ and $(\tau, \varphi)$ are standard, it is straightforward to show that $\left(\tau^{*}, \varphi^{*}\right)$ does not strictly Pareto-dominate $(\tau, \varphi)$.

We begin by proving (I). First, suppose $(\tau, \varphi)$ is not Pareto-dominated by another such rule. By Claim 1, its standardization $\left(\tau^{\oplus}, \varphi^{\oplus}\right)$ is not Pareto-dominated by another such rule. By Claim 2, $\left(\tau^{\oplus}, \varphi^{\oplus}\right)$ is unrelenting. By Claim 3, $\left(\tau^{\oplus}, \varphi^{\oplus}\right)$ has marginal cost award profile in $\mathcal{F}$. Since $(\tau, \varphi)$ and its standardization share a social-dividend schedule, altogether, $(\tau, \varphi)$ has an unrelenting social-dividend schedule whose marginal cost award profile is in $\mathcal{F}$, as desired.

Second, suppose $(\tau, \varphi)$ has an unrelenting social-dividend schedule whose marginal cost award profile is in $\mathcal{F}$. Assume, by way of contradiction, $\left(\tau^{\prime}, \varphi^{\prime}\right)$ is a rule satisfying the axioms of (I) that strictly Pareto-dominates $(\tau, \varphi)$. Let $a \in \mathcal{A}$ be the marginal cost award profile of $(\tau, \varphi)$, let $a^{\prime} \in \mathcal{A}$ be the marginal cost award profile of $\left(\tau^{\prime}, \varphi^{\prime}\right)$, let $\left(\tau^{\oplus}, \varphi^{\oplus}\right)$ be the standardization of $(\tau, \varphi)$, let $\left(\tau^{\prime \oplus}, \varphi^{\prime \oplus}\right)$ be the standardization of $\left(\tau^{\prime}, \varphi^{\prime}\right)$, and let $\left(\tau^{*}, \varphi^{*}\right)$ be the standard citizen-shareholder rule with the unrelenting social-dividend schedule whose marginal cost award profile is $a^{\prime}$. By Claim $1,\left(\tau^{\prime \oplus}, \varphi^{\prime \oplus}\right)$ strictly Paretodominates $(\tau, \varphi)$. By Claim $2,\left(\tau^{*}, \varphi^{*}\right)$ Pareto-dominates $\left(\tau^{\prime \oplus}, \varphi^{\prime \oplus}\right)$, so $\left(\tau^{*}, \varphi^{*}\right)$ strictly Pareto-dominates $(\tau, \varphi)$. But then by Claim 3, $a^{\prime}>a$, contradicting $a \in \mathcal{F}$.

The proof of (II) is analogous.

\section{References}

Arrow, L. And Debreu, G. (1954). "Existence of an Equilibrium for a Competitive Economy." Econometrica 22, 265-290.

Ashlagi, I. AND Serizawa, S. (2012). "Characterizing Vickrey allocation rule by anonymity." Social Choice and Welfare 38, 531-542. 
Atlamaz, M. And Yengin, D. (2008). "Fair Groves mechanisms." Social Choice and Welfare 31, 573-587.

Attiyeh, G., Franciosi, R., And Isaac, R. (2000). "Experiments with the pivot process for providing public goods." Public Choice 102, 95-114.

Bardhan, P. And Roemer, J. (1992). "Market Socialism: A Case for Rejuvenation." Journal of Economic Perspectives 6, 101-116.

Baumol, W . (1977). "On the Proper Cost Tests for Natural Monopoly in a Multiproduct Industry." The American Economic Review 67, 809-822.

BöHm-BAwerk, E. (1888). Kapital und Kapitalzins. Zweite Abteilung: Positive Theorie des Kapitales [in German]. Innsbruck, Austria: Verlag der Wagner'schen UniversitätsBucchandlung. Translation: Böhm-Bawerk, E. (1891). The Positive Theory of Capital. Translator: Smart, W. London, England: Macmillan and Co.

Buchanan, J. (1965). "An Economic Theory of Clubs." Economica 32, 1-14.

Chew, S. And Serizawa, S. (2007). "Characterizing the Vickrey combinatorial auction by induction." Economic Theory 33, 393-406.

Clarke, E. (1971). "Multipart Pricing of Public Goods." Public Choice 8, 19-33.

Deb, R. And Razzolini, L. (1999a). "Voluntary cost sharing for an excludable public project." Mathematical Social Sciences 37, 123-138.

Deb, R. And Razzolini, L. (1999b). "Auction-Like Mechanisms for Pricing Excludable Public Goods." Journal of Economic Theory 88, 340-368.

Demange, G. (1982). "Strategyproofness in the Assignment Market Game." Mimeo. Laboratoire d'Econometrie de l'Ecole Polytechnique, Paris, France.

Demange, G. And Gale, D. (1985). "The Strategy Structure of Two-Sided Matching Markets." Econometrica 53, 873-888.

Foley, D. (1967). "Resource allocation and the public sector." Yale Economic Essays $7,45-98$.

Friedman, M. (1962). Capitalism and Freedom. Chicago, Illinois: The University of Chicago Press.

Gale, D. (1960). The Theory of Linear Economic Models. New York, New York: McGraw-Hill Book Company.

GiBBARD, A. (1973). "Manipulation of voting schemes: a general result." Econometrica $41,587-601$.

Green, J. And Laffont, J. (1977). "Characterization of Satisfactory Mechanisms for the Revelation of Preferences for Public Goods." Econometrica 45, 727-738.

Green, J. And Laffont, J. (1979). Incentives in Public Decision Making. Amsterdam, the Netherlands: North Holland Publishing Company. 
Groves, T. (1973). "Incentives in Teams." Econometrica 41, 617-631.

Groves, T. AND LeDYARD, J. (1987). "Incentive compatibility since 1972." In: Information, Incentives, and Economic Mechanisms. Editors: Groves, T., Radner, R., and Reiter, S. Minneapolis, Minnesota: University of Minnesota Press.

Gul, F. And Stacchetti, E. (1999). "Walrasian Equilibrium with Gross Substitutes." Journal of Economic Theory 87, 95-124.

Guo, M., Markakis, E., Apt, K., And Conitzer, V. (2013). "Undominated Groves Mechanisms." Journal of Artificial Intelligence Research 46, 129-163.

Hashimoto, K. And SAitoh, H. (2016). "Strategy-proof rules for an excludable public good." Social Choice and Welfare 46, 749-766.

Hayek, F. (1945). "The Use of Knowledge in Society." The American Economic Review $35,519-530$.

Holmström, B. (1979). "Groves' Scheme on Restricted Domains." Econometrica 47, 1137-1144.

Hurwicz, L. (1972). "On informationally decentralized systems." In: Decision and Organization: A Volume in Honor of Jacob Marschak. Editors: McGuire, C. and Radner, R. Amsterdam, the Netherlands: North-Holland Publishing Company.

JuAREz, R. (2013). "Group strategyproof cost sharing: The role of indifferences." Games and Economic Behavior 82, 218-239.

JuAREz, R. "Optimal group strategyproof cost sharing." Working paper, 2013.

Kagel, J., Harstad, R., And Levin, D. (1987). "Information impact and allocation rules in auctions with affiliated private values: A laboratory study." Econometrica 55, 1275-1304.

Kagel, J. And Levin, D. (1993). "Independent private value auctions: Bidder behavior in first-, second-, and third-price auctions with varying numbers of bidders." Economic Journal 103, 868-879.

Kawagoe, T. And Mori, T. (2001). "Can the pivotal mechanism induce truth-telling? An experimental study." Public Choice 108, 331-354.

Kelso, A. And Crawford, V. (1982). "Job Matching, Coalition Formation, and Gross Substitutes." Econometrica 50, 1483-1504.

Kolm, S. (1971). Justice et Equité. Paris, France: Centre d'Etudes Prospectives et d'Economie Mathématique Appliquées à la Planification (CEPREMAP).

Koopmans, T. and Beckmann, M. (1957). "Assignment Problems and the Location of Economic Activities." Econometrica 25, 53-76.

Lange, O. (1936). "On the Economic Theory of Socialism: Part One." The Review of Economic Studies 4, 53-71. 
Ledyard, J. And Roberts, J. (1975). "On the incentive problem with public goods." Manuscript.

LeOnard, H. (1983). "Elicitation of Honest Preferences for the Assignment of Individuals to Positions." The Journal of Political Economy 91, 461-479.

Lerner, A. (1944). The Economics of Control: Principles of Welfare Economics. New York, New York: The Macmillan Company.

Leroux, J. (2004). "Strategy-proofness and efficiency are incompatible in production economies." Economic Letters 83, 335-340.

LI, S. (2016). "Obviously Strategy-Proof Mechanisms." Working paper.

Lindahl, E. (1919). Die Gerechtigkeit der Besteurung: Eine Analyse der Steuerprinzipien auf Grundlage der Grenznutzentheorie [in German]. Lund, Sweden: Gleerupska Universitets-Bokhandeln. Partial Translation: Lindahl, E. "Just Taxation-A Positive Solution." In: (1958). Classics in the Theory of Public Finance. Editors: Musgrave, R. and Peacock, A. London, England: Palgrave Macmillan.

McKenzIE, L. (1954). "On Equilibrium in Graham's Model of World Trade and Other Competitive Systems." Econometrica 22, 147-161.

Maniquet, F. And Sprumont, Y. (1999). "Efficient Strategy-proof Allocation Functions in Linear Production Economies." Economic Theory 14, 583-595.

Marshall, A. (1890). Principles of Economics. London, England: Macmillan and Company, Limited.

Massó, Nicolò, Sen, Sharma, And Ülkü. "On Equal Cost Sharing in the Provision of an Excludable Public Good." Working paper, 2015.

von Mises, L. (1920). "Economic Calculation in the Socialist Commonwealth." In: Collectivist Economic Planning: Critical Studies on the Possibilities of Socialism. Editor: Hayek, F. London, England: George Routledge \& Sons. Pages 87-130.

Morimoto, S. And Serizawa, S. (2015). "Strategy-proofness and efficiency with nonquasi-linear preferences: A characterization of minimum price Walrasian rule." Theoretical Economics 10, 445-487.

Moulin, H. (1986). "Characterizations of the pivotal mechanism." Journal of Public Economics 31, 53-78.

Moulin, H. And Shenker, S. (2001). "Strategyproof sharing of submodular costs: budget balance versus efficiency." Economic Theory 18, 511-533.

Mutuswami, S. (2004). "Strategyproof cost sharing of a binary good and the egalitarian solution." Mathematical Social Sciences 48, 271-280.

Myerson, R. (1981). "Optimal Auction Design." Mathematics of Operation Research 6, 58-73. 
Niederle, M. And Roth, A. (2003). "Unraveling Reduces Mobility in a Labor Market: Gastroenterology with and without a Centralized Match." Journal of Political Economy $111,1342-1352$.

Ohseto, S. (2000). "Characterizations of Strategy-Proof Mechanisms for Excludable versus Nonexcludable Public Projects." Games and Economic Behavior 32, 51-66.

Oнseto, S. (2004). "Implementing egalitarian-equivalent allocation of indivisible goods on restricted domains." Economic Theory 23, 659-670.

Ohseto, S. (2005). "Augmented serial rules for an excludable public project." Economic Theory 26, 589-606.

Ohseto, S. (2006). "Characterizations of strategy-proof and fair mechanisms for allocating indivisible goods." Economic Theory 29, 111-121.

OHsEto, S. (2009). " $\alpha$-serial mechanisms for the provision of an excludable public good." The Japanese Economic Review 61, 507-516.

PÁpAI, S. (2003). "Groves sealed bid auctions of heterogeneous objects with fair prices." Social Choice and Welfare 20, 371-385.

Pazner, E. And Schmeidler, D. (1978). "Egalitarian Equivalent Allocations: A New Concept of Economic Equity." The Quarterly Journal of Economics 92, 671-687.

Piccione, M. And Rubinstein, A. (2007). "Equilibrium in the Jungle." Economic Journal 117, 883-896.

Porter, R., Shoham, Y., And Tennenholtz, M. (2004). "Fair imposition." Journal of Economic Theory 118, 209-228.

Rawls, J. (1971). A Theory of Justice. Cambridge, Massachusetts: Belknap Press.

Roberts, K. (1979). "The characterization of implementable choice rules." In: Aggregation and Revelation of Preferences. Editor: Laffont, J. Amsterdam, the Netherlands: North Holland Publishing Company.

Saijo, T., Sjöström, T., And Yamato, T. (2007). "Secure implementation." Theoretical Economics 2, 203-229.

SAitoh, H. AND SERIZAWA, S. (2008). "Vickrey allocation rule with income effect." Economic Theory 35, 391-401.

SAKAI, T. (2008). "Second price auctions on general preference domains: two characterizations." Economic Theory 37: 347-356.

Samuelson, P. (1954). "The Pure Theory of Public Expenditure." The Review of Economics and Statistics 36, 387-389.

Schmeidler, D. And Vind, K. (1972). "Fair net trades." Econometrica 40, 637-642.

Serizawa, S. (2002). "Inefficiency of Strategy-Proof Rules for Pure Exchange Economies." Journal of Economic Theory 106, 219-241. 
SERIZAWA, S. And Weymark, J. (2003). "Efficient strategy-proof exchange and minimum consumption guarantees." Journal of Economic Theory 109, 246-263.

Shapley, L. And Shubik, M. (1972). "The Assignment Game I: The Core." International Journal of Game Theory 1, 111-130.

Steinhaus, H. (1948). "The problem of fair division." Econometrica 16, 101-104.

Svensson, L. (1983). "Large Indivisibilities: An Analysis with Respect to Price Equilibrium and Fairness." Econometrica 51, 939-954.

Thomson, W. (1983). "The Fair Division of a Fixed Supply Among a Growing Population." Mathematics of Operations Research 8, 319-326.

Tinbergen, J. (1946). Redelijke Inkomensverdeling [Reasonable Income, in Dutch]. Haarlem, the Netherlands: De Gulden Pers.

Velez, R. (2016). "Fairness and externalities." Theoretical Economics 11, 381-410.

ViCKrey, W. (1961). "Counterspeculation, Auctions, and Competitive Sealed Tenders." The Journal of Finance 16, 8-37.

WALras, L. (1896). Éléments d'économie politique pure ou théorie de la richesse sociale [In French]. Lausanne, Switzerland: L. Corbaz \& Company. English translation: Jaffé, W. (1954) Elements of pure economics, or the theory of social wealth. London, England: George Allen and Unwin.

WiCKSELl, K. (1896). Finanztheoretische Untersuchungen nebst Darstellung und Kritik des Steuerwesens Schwedens [Financial theory with presentation and criticism of taxation in Sweden, in German]. Jena, Germany: Verlag von Gustav Fischer. English translation: In: (1958) Classics in the theory of public finance. Editors: Musgrave, R. and Peacock, A. London, United Kingdom: Macmillan Publishers Ltd.

Yengin, D. (2012a). "Egalitarian-equivalent Groves mechanisms in the allocation of heterogeneous objects." Social Choice and Welfare 38, 137-160.

Yengin, D. (2012b). "Characterizing Welfare-egalitarian Mechanisms with Solidarity When Valuations are Private Information." The B.E. Journal of Theoretical Economics. 12.

Yengin, D. (2013a). "Population monotonic and strategy-proof mechanisms respecting welfare lower bounds." Journal of Mathematical Economics 49, 389-397.

Yengin, D. (2013b). "Identical preferences lower bound for allocation of heterogeneous tasks and NIMBY problems." Journal of Public Economic Theory 15, 580-601.

YENGIN, D. (2016). "No-envy and egalitarian-equivalence under multi-object-demand for heterogeneous objects." Social Choice and Welfare, forthcoming.

Yu, Y. (2007). "Serial cost sharing of an excludable public good available in multiple units." Social Choice and Welfare 29, 539-555. 